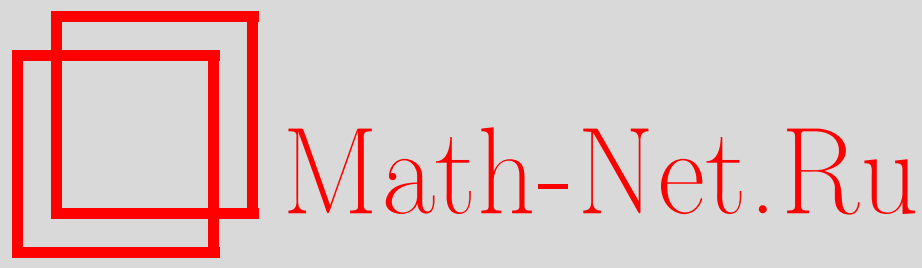

В. В. Пупышев, Длина и эффективный радиус двумерного рассеяния квантовой частицы центральным короткодействующим потенциалом, ТМФ, 2014, том 180, номер 3, 342-367

DOI: https://doi.org/10.4213/tmf8582

Использование Общероссийского математического портала Math-Net.Ru подразумевает, что вы прочитали и согласны с пользовательским соглашением http://www . mathnet.ru/rus/agreement

Параметры загрузки:

IP : 34.227 .88 .159

26 апреля 2023 г., 13:31:48

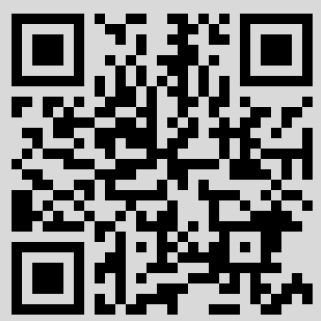




\title{
ФИЗИКА
}

Том 180, № 3

сентябрь, 2014

\section{ДЛИНА И ЭФФЕКТИВНЫЙ РАДИУС ДВУМЕРНОГО РАССЕЯНИЯ КВАНТОВОЙ ЧАСТИЦЫ ЦЕНТРАЛЬНЫМ КОРОТКОДЕЙСТВУЮЩИМ ПОТЕНЦИАЛОМ}

\begin{abstract}
Дан вывод и качественный анализ нелинейных и линейных уравнений, предназначенных для вычисления длины рассеяния и эффективного радиуса. Найдены и исследованы точные решения этих уравнений в случае центрального потенциала прямоугольной формы. Выявлена связь между эффективным радиусом и длиной рассеяния. Особое внимание уделено случаям нулевой и неограниченной длин рассеяния.
\end{abstract}

Ключевые слова: двумерное рассеяние, короткодействующий потенциал, длина рассеяния, эффективный радиус.

DOI: $10.4213 / \operatorname{tmf} 8582$

\section{1. ВВЕДЕНИЕ}

Понятия длины рассеяния и эффективного радиуса широко применяются в теории трехмерного потенциального рассеяния [1]-[5]. В ядерную физику низких энергий [6] эти понятия ввел Бете [7].

В настоящее время развитие теории двумерного потенциального рассеяния стимулируется выдающимися успехами экспериментальной физики ультрахолодных газов в магнитооптических ловушках различных конфигураций [8]-[11], в частности в дискообразных ловушках. Экспериментально достижимая температура газа настолько мала, что в такой ловушке длина де Бройля [1] частицы газа сравнима с поперечным размером диска-ловушки. Поэтому квантовое движение частиц газа в основном происходит в плоскости, проходящей через края диска. Изменяя внешнее электромагнитное поле, создающее такую ловушку, удается удерживать в ней заданное четное число атомов и управлять парными взаимодействиями между ними. Минимальное число атомов, запертых в диске-ловушке и движущихся в плоскости, равно двум [11]. В системе центра масс таких ультрахолодных атомов квантово-

* Объединенный институт ядерных исследований, Дубна, Московская обл., Россия. E-mail: pupyshev@theor.jinr.ru 
механическая задача об их относительном движении сводится к задаче о медленном движении одной квантовой частицы в двумерной плоскости. Длина рассеяния и эффективный радиус являются универсальными (не зависящими от формы потенциала) характеристиками такого движения. Поэтому их исследование представляется актуальной задачей современной теории двумерного рассеяния.

Длина и эффективный радиус двумерного рассеяния квантовой частицы центральным короткодействующим потенциалом были исследованы многими авторами (см., например, работы [5], [12]-[21]). Тем не менее квантово-механический анализ этих фундаментальных характеристик низкоэнергетического рассеяния далек от завершения. В частности, не выведены уравнения для функций, обладающих прозрачным физическим смыслом и определяющих значения длины рассеяния и эффективного радиуса; открытыми остаются вопросы о связи эффективного радиуса с длиной рассеяния и о переопределении этих параметров в случаях их нулевых или бесконечно больших по модулю значений; ни для какого потенциала неизвестны представления длины рассеяния и эффективного радиуса через элементарные или специальные функции.

Главная цель настоящей работы - восполнить эти недостатки современной теории двумерного рассеяния. В разделе 2 собраны основные определения. В разделе 3 дан вывод и качественный анализ уравнений для вспомогательных функций, значения которых в пределе их бесконечно большого аргумента являются длиной рассеяния и эффективным радиусом. В разделе 4 найдены и исследованы точные решения полученных уравнений в случае потенциала прямоугольной формы. В разделе 5 эти же уравнения использованы для расчета длины и эффективного радиуса двумерного рассеяния нейтрона на протоне в случае ${ }^{1} S_{0}$-потенциала Рида с мягким кором [6]. В разделе 6 исследованы случаи нулевой и неограниченно большой по модулю длины рассеяния и в этих случаях предложены новые определения длины рассеяния и эффективного радиуса. Основные результаты, полученные в разделах $3-6$, перечислены в заключении.

\section{2. ОСНОВНЫЕ ПОЯСНЕНИЯ И ОПРЕДЕЛЕНИЯ}

Начнем с физических предположений. Предположим, что квантовая частица $p_{1}$ имеет массу $m_{1}$ и движется лишь в двумерной плоскости $\mathcal{P}$ ее координатного пространства $\mathbb{R}^{3}$. Считаем, что некоторая неподвижная точка $O$ этой плоскости является силовым центром, воздействующим на частицу $p_{1}$ посредством потенциала $V$, который зависит только от обезразмеренного расстояния $x$ между точкой $O$ и этой частицей и подчинен следующим условиям:

$$
V(x) \in C^{0}(0, \infty), \quad \lim _{x \rightarrow 0} x^{2}|V(x)|=0, \quad \lim _{x \rightarrow \infty} x^{n}|V(x)|=0, \quad n=0,1,2, \ldots .
$$

Такой потенциал принято называть центральным, слабосингулярным в нуле, непрерывным на всей полуоси $x>0$ и короткодействующим [5]. Отметим, что условие непрерывности можно ослабить: в области $0<x<\infty$ потенциал $V(x)$ может быть кусочно-непрерывной функцией с конечным числом точек разрыва первого рода. Пример такой функции - потенциал прямоугольной формы $V(x)=V_{0} \theta(b-x)$, где константы $V_{0} \neq 0$ и $b>0$, а $\theta(b-x)$ - функция Хевисайда, равная единице при $x \leqslant b$ и нулю в противном случае. 
Теперь перечислим известные в теории двумерного рассеяния [5], [21]-[23] следствия сделанных выше предположений о потенциале. В поле центрального потенциала $V(x)$ полный набор квантовых чисел частицы $p_{1}$ состоит из ее обезразмеренного волнового числа $q$ и дискретного числа $\lambda$, принимающего любые полуцелые значения начиная с $-1 / 2$. При условиях (1) радиальная волновая функция $u_{\lambda}(x ; q)=\langle x \mid q, \lambda\rangle$ состояния рассеяния $|q, \lambda\rangle, q>0$, определяется как регулярное (ограниченное на всей полуоси $x>0$ ) решение одномерного уравнения Шредингера

$$
\left[\partial_{x}^{2}+q^{2}-\lambda(\lambda+1) x^{-2}-V(x)\right] u_{\lambda}(x ; q)=0, \quad x>0,
$$

с начальным условием

$$
u_{\lambda}(x ; q) \sim(q x)^{\lambda+1}, \quad q x \rightarrow 0
$$

и асимптотикой

$$
u_{\lambda}(x ; q) \rightarrow \sin \left(q x-\frac{\pi \lambda}{2}+\delta_{\lambda}(q)\right), \quad \frac{q x}{|\lambda|} \rightarrow \infty .
$$

Величины $\delta_{\lambda}(q)$ и $\sigma_{\lambda}(q)$ называются парциальными фазой и сечением упругого рассеяния в состоянии $|q, \lambda\rangle$. Сечение $\sigma_{\lambda}(q)$ вычисляется по формулам [5], [21]

$$
\sigma_{\lambda}(q) \equiv \frac{\sigma_{\lambda}^{\mathrm{u}}(q)}{\operatorname{ctg}^{2} \delta_{\lambda}(q)+1}, \quad \sigma_{\lambda}^{\mathrm{u}}(q) \equiv \frac{4}{q}\left(2-\delta_{2 \lambda,-1}\right) .
$$

Здесь и далее $\delta_{i, j}-$ символ Кронекера. При любом $q \geqslant 0$ сечение $\sigma_{\lambda}(q)$ не превышает своего унитарного предела $\sigma_{\lambda}^{\mathrm{u}}(q)$. Предел $q \rightarrow+0$ называется пределом низких энергий.

В случае центрального короткодействующего потенциала физические и математические причины, обуславливающие существенное отличие двумерного рассеяния от трехмерного, детально обсуждались в работах [5], [12]-[26], причем разными способами.

Предложим еще один подход к выявлению этих причин. Сначала с помощью формулы $\ell=\lambda+1 / 2$ каждому полуцелому значению квантового числа $\lambda=-1 / 2,1 / 2, \ldots$ поставим в соответствие целое число $\ell=0,1, \ldots$ Далее заметим, что после замены $\lambda \mapsto \ell$ краевая задача Шредингера (2)-(4) становится краевой задачей для радиальной волновой функции $u_{\ell}(x ; q)$ трехмерного рассеяния квантовой частицы $p_{1}$ потенциалом $V(x)$ в состоянии $|q, \ell\rangle$ с волновым числом $q$ и угловым моментом $\ell$ (см. монографию [3]). Положим

$$
\begin{aligned}
V_{\lambda}(x) & \equiv \frac{\lambda(1+\lambda)}{x^{2}}, & V_{\ell}(x) & \equiv \frac{\ell(1+\ell)}{x^{2}}, \\
V(x ; \lambda) & \equiv V(x)+V_{\lambda}(x), & V(x ; \ell) & \equiv V(x)+V_{\ell}(x) .
\end{aligned}
$$

Согласно радиальному уравнению Шредингера (2) можно считать, что двумерное или трехмерное рассеяние квантовой частицы $p_{1}$ в состояниях $|q, \lambda\rangle$ или $|q, \ell\rangle$ порождается соответствующими эффективными потенциалами $V(x, \lambda)$ или $V(x, \ell)$. Сравним эти потенциалы и их компоненты. 
При значении $\lambda=-1 / 2$ притягивающему (центростремительному) потенциалу $V_{\lambda}(x)=-1 /(2 x)^{2}$ в трехмерном, а именно $S$-волновом $(\ell=0)$, рассеянии соответствует тождественно равный нулю потенциал $V_{\ell}(x) \equiv 0$. Для всех остальных значений квантовых чисел $\lambda \geqslant 1 / 2$ и $\ell=\lambda+1 / 2$ оба потенциала $V_{\lambda}(x)$ и $V_{\ell}(x)$ являются отталкивающими (центробежными) потенциалами и подчиняются неравенству $V_{\lambda}(x)<V_{\ell}(x)$ при $x>0$. Это неравенство и определения (6) порождают соотношения $V(x, \lambda)<V(x, \ell)$ для $x>0$, справедливые при любом потенциале $V(x)$ и любом значении $\lambda \geqslant-1 / 2$. Вследствие этого воздействие одного и того же потенциала $V(x)$ на движение квантовой частицы $p_{1}$ оказывается более сильным в состоянии двумерного рассеяния $|q, \lambda\rangle$, чем в состоянии трехмерного рассеяния $|q, \ell\rangle$. Этот вывод можно сформулировать иначе: если $2 \lambda \geqslant 1$, то потенциал $V(x)$ в двумерном рассеянии экранируется потенциалом $V_{\lambda}(x)$ гораздо слабее, чем потенциалом $V_{\ell}(x)$ в трехмерном рассеянии.

Отмеченный факт имеет три физически важных следствия, которые доказаны в теории дифференциальных уравнений [27] как теоремы об оценках. Сформулируем первое следствие: по крайней мере в области $0<x<b, b \ll 1$, небольших расстояний выполняется соотношение $\left|u_{\lambda}(x ; q)\right|^{2}>\left|u_{\ell}(x ; q)\right|^{2}$. Оно означает, что при любом потенциале $V(x)$ вероятность обнаружить квантовую частицу $p_{1}$ в двумерном круге $x \leqslant b$ с центром $O$ больше, чем вероятность нахождения этой же частицы в трехмерном шаре $x \leqslant b$ с тем же центром. Обсудим второе следствие. Пусть в поле некоторого (необязательно короткодействующего) потенциала $V(x)$ квантовая частица $p_{1}$ в пространстве $\mathbb{R}^{3}$ имеет связанные состоянии $\left|i Q_{n}, \ell\right\rangle, Q_{n}>0$, $n=1,2, \ldots, N$, с энергиями связи $B_{n}=Q_{n}^{2}$, причем $B_{1}>B_{2}>\cdots>B_{N}$. Тогда эта частица в плоскости $\mathcal{P}$ также будет иметь связанные состояния $\left|i q_{n}, \lambda\right\rangle, q_{n}>0$, $\lambda=\ell-1 / 2$, с энергиями связи $b_{n}=q_{n}^{2}, b_{1}>b_{2}>\cdots>b_{N}$, причем $b_{n}>B_{n}$ для всех $n=1,2, \ldots N$. Третье следствие звучит так: если при данных $V(x)$ и $\ell$ в пространстве $\mathbb{R}^{3}$ квантовая частица $p_{1}$ не имеет связанных состояний, то при том же потенциале и при $\lambda=\ell-1 / 2$ эта частица в плоскости $\mathcal{P}$ может иметь связанные состояния.

Приведем физически интересные примеры указанных выше следствий.

ПримеР 1. Пусть $V(x)=1 / x$ - отталкивающий кулоновский потенциал, $\lambda=-1 / 2$ и $\ell=0$. Тогда имеют место асимптотики [17], [18]

$$
\left|u_{-1 / 2}(x ; q)\right|^{2} \sim C_{-1 / 2}^{2}(\eta) x, \quad\left|u_{0}(x ; q)\right|^{2} \sim C_{0}^{2}(\eta) x^{2}, \quad x \rightarrow 0,
$$

в которых кулоновские барьерные множители определены формулами

$$
C_{-1 / 2}^{2}(\eta) \equiv \frac{\pi}{e^{2 \pi \eta}+1}, \quad C_{0}^{2}(\eta) \equiv \frac{2 \pi \eta}{e^{2 \pi \eta}-1}, \quad \eta=\frac{1}{2 q} .
$$

Следовательно, верны неравенства

$$
\begin{gathered}
\left|u_{-1 / 2}(x ; q)\right|^{2} \gg\left|u_{0}(x ; q)\right|^{2}, \quad x \ll 1, \\
C_{-1 / 2}^{2}(\eta) \ll C_{0}^{2}(\eta), \quad q \ll 1 .
\end{gathered}
$$

Предположим, что в точке $O$ находится ядро $A$, а наша квантовая частица $p_{1}$ тоже является некоторым ядром таким, что возможна экзотермическая реакция слияния 
ядер $A$ и $p_{1}$ при низкой энергии $q^{2}$ их столкновения в поле кулоновского отталкивающего потенциала $V(x)=1 / x$. В силу приведенных выше неравенств вероятность такой реакции в плоскости $\mathcal{P}$ будет намного больше, чем в $\mathbb{R}^{3}$.

ПримеР 2. Пусть $V(x)=-1 / x-$ притягивающий кулоновский потенциал. Тогда при одном и том же главном квантовом числе $n=1,2, \ldots$ энергии связи $B_{n}$ и $b_{n}$ квантовой частицы $p_{1}$ в пространстве $\mathbb{R}^{3}$ и в плоскости $\mathcal{P}$ определяются формулой [1] $B_{n}=n^{-2}$ и формулой [28] $b_{n}=(n-1 / 2)^{-2}$. Следовательно, $b_{n}>B_{n}$ при любом $n$. Предположим, что в точке $O$ расположен протон $p$, частица $p_{1}$ является электроном $e^{-}$, а взаимодействие в системе $\left\{e^{-}, p\right\}$ исчерпывается кулоновским притяжением $V(x)=-1 / x$. Положив $\lambda=-1 / 2, \ell=0$ и $n=1$, убеждаемся в том, что энергии связи $b_{1}$ и $B_{1}$ основного состояния системы $\left\{e^{-}, p\right\}$ в $\mathcal{P}$ и в $\mathbb{R}^{3}$ связаны равенством $b_{1}=4 B_{1}$.

ПримеР 3. Как известно [24], в случае $2 \lambda=-1$ при любом сколь угодно слабом, но притягивающем короткодействующем потенциале $V(x)$ квантовая частица $p_{1}$ в плоскости $\mathcal{P}$ имеет хотя бы одно связанное состояние. В пространстве $\mathbb{R}^{3}$ такой потенциал не порождает ни одного связанного состояния.

Особый случай $2 \lambda=-1$ стоит обсудить более подробно. В этом случае значение $1 / 4$ константы центростремительного потенциала $V_{\lambda}(x)=-(1 / 4) x^{-2}$ является критическим в следующем смысле: если ее увеличить на сколь угодно малое, но положительное число, то спектр оператора Шредингера уравнения (2) станет неограниченным снизу и будет возможным падение [1] квантовой частицы $p_{1}$ в плоскости $\mathcal{P}$ на силовой центр $О$. Известным следствием [1], [12] наличия такого критического значения является существенная роль потенциала $-(1 / 4) x^{-2}$ в пределе $q \rightarrow 0+0$. В этом пределе для любого короткодействующего потенциала $V(x)$ старшие слагаемые асимптотик фазы рассеяния $\delta_{-1 / 2}(q)$ и соответствующего ей сечения (5) неограниченно возрастают: $\delta_{-1 / 2}(q) \sim(2 / \pi) \ln q$ и $\sigma_{-1 / 2}(q) \sim q^{-1}(\pi / \ln q)^{2}$. Для сравнения напомним, что в этом же пределе фаза и сечение $S$-волнового трехмерного рассеяния $\delta_{0}(q)$ и $\sigma_{0}(q)$ сходятся соответственно к произведениям $-a q$ и $4 \pi a^{2}$, которые зависят от потенциала $V(x)$ посредством конечной константы $a$, называемой длиной $S$-волнового рассеяния [1]-[6].

Теперь поясним, почему фазы двумерного и трехмерного рассеяния $\delta_{\lambda}(q)$ и $\delta_{\ell}(q)$ имеют существенно разную функциональную зависимость от волнового числа $q$.

Обсуждение фазы двумерного рассеяния начнем со случая $V(x) \equiv 0$ при $x \geqslant 0$. В этом случае краевая задача (2)-(4) имеет два линейно независимых решения $j_{\lambda}(\rho)$ и $\tilde{n}_{\lambda}(\rho), \rho \equiv q x$, связанных с функциями Бесселя $J_{m}(\rho)$ и $Y_{m}(\rho)$ целого порядка $m$ формулами

$$
j_{\lambda}(\rho)=\sqrt{\frac{\pi \rho}{2}} J_{m}(\rho), \quad \tilde{n}_{\lambda}(q x)=\sqrt{\frac{\pi \rho}{2}} Y_{m}(\rho), \quad m=\lambda+\frac{1}{2} .
$$

Как известно [29], функция $J_{m}(\rho)$ может быть представлена как бесконечный ряд по целым степеням ее аргумента, а функция $Y_{m}(\rho)$ - как бесконечный ряд, первое слагаемое которого

$$
\frac{2}{\pi}\left(\ln \frac{\rho}{2}+\gamma\right) J_{m}(\rho)=\frac{2}{\pi} \ln x J_{m}(\rho)+h(q) J_{m}(\rho)
$$


содержит константу Эйлера $\gamma$ и логарифмическую функцию $h(q)$ волнового числа $q$,

$$
h(q) \equiv \frac{2}{\pi}\left(\ln \frac{\rho}{2}+\gamma\right)=\frac{2}{\pi} \ln \frac{q}{q_{0}}, \quad q_{0} \equiv 2 e^{-\gamma}=1.122918 \ldots .
$$

Следовательно, разложения функций $Y_{m}(\rho)$ и $\tilde{n}_{\lambda}(\rho)$ содержат функцию $h(q)$.

Рассмотрим случай финитного потенциала. Предположим, что короткодействующий потенциал $V(x)$ отличен от нуля лишь на отрезке $0 \leqslant x \leqslant b, b<\infty$, и решение $u_{\lambda}(x ; q)=u^{-}(x ; q)$ задачи $(2),(3)$ известно на этом же отрезке. При условиях (1) в соответствии с теорией Фукса [27] такое решение может содержать только полуцелые степени параметра $q$. В области $x>b$ общее решение задачи $(2),(4)$ содержит фазу рассеяния $\delta_{\lambda}(q)$ и имеет вид

$$
u_{\lambda}(x ; q)=u^{+}(x ; q) \equiv j_{\lambda}(x ; q) \cos \delta_{\lambda}(q)-\tilde{n}_{\lambda}(x ; q) \sin \delta_{\lambda}(q) .
$$

Условие "сшивки" [1], [5] логарифмических производных функций $u^{ \pm}(x, q)$ в точке $x=b$ дает представление котангенса фазы рассеяния $\delta_{\lambda}(q)$ через функции $u^{-}(b, q)$, $j_{\lambda}(b q)$ и $\tilde{n}_{\lambda}(b q)$ аргумента $q$. Используя это представление, нетрудно доказать, что в рассмотренном случае финитного потенциала с конечным носителем $0 \leqslant x \leqslant b$ при любом $\lambda$ функция $\operatorname{ctg} \delta_{\lambda}(q)$ является суммой двух слагаемых, одно из которых содержит логарифмическую функцию $h(q)$, порожденную разложением функции $\tilde{n}_{\lambda}(b q)$, а второе является бесконечным рядом $S_{\lambda}(q)$ по полуцелым степеням аргумента $q$.

Такое же утверждение для любого необязательно финитного, но короткодействующего потенциала доказано для случая $2 \lambda=-1$ в работах [14]--[19] и в общем случае $2 \lambda \geqslant-1$ в работах [20], [21].

Перейдем к обсуждению фазы $\delta_{\ell}(q)$ трехмерного рассеяния. В задаче Шредингера сделаем замену $\lambda \mapsto \ell=\lambda+1 / 2$. В случае $V(x) \equiv 0$ линейно независимыми решениями $j_{\ell}(q x)$ и $n_{\ell}(q x)$ получившейся задачи будут известные сферические функции Риккати-Бесселя [29]. Такие функции являются бесконечными рядами по целым степеням аргумента $\rho$. Как известно [3], [5], из этого факта следует, что для любого короткодействующего потенциала и при любом $\ell=0,1, \ldots$ котангенс фазы $\delta_{\ell}(q)$ трехмерного рассеяния есть бесконечный ряд $S_{\ell}(q)$ по целым степеням ее аргумента $q$, а функция эффективного радиуса $K^{\mathrm{s}}(q)$ - бесконечный ряд по четным степеням этого же аргумента:

$$
K^{\mathrm{s}}(q) \equiv q^{2 \ell+1} \operatorname{ctg} \delta_{\ell}(q)=-\frac{1}{a^{\mathrm{s}}}+\frac{q^{2}}{2} r_{\mathrm{eff}}^{\mathrm{s}}+\cdots
$$

Напомним определения длины и эффективного радиуса трехмерного рассеяния. В теории трехмерного рассеяния [1]-[5], в ядерной [6], атомной и молекулярной физике длиной и эффективным радиусом трехмерного рассеяния квантовой частицы $p_{1}$ центральным короткодействующим потенциалом в состоянии $|q, \ell\rangle$ принято называть коэффициенты $a^{\mathrm{s}}$ и $r_{\mathrm{eff}}^{\mathrm{s}}$ разложения функции эффективного радиуса $K^{\mathrm{s}}(q)$.

Теперь перечислим все известные к настоящему времени определения длины рассеяния и эффективного радиуса для двумерного рассеяния квантовой частицы $p_{1}$ центральным короткодействующим потенциалом в состоянии $|q, \lambda\rangle$.

В работах [12], [13] использовались понятия дифференциальной и полной длин рассеяния. Дифференциальной длиной рассеяния в этих работах назван квадрат 
модуля амплитуды двумерного рассеяния, а полной длиной рассеяния - интеграл от такого квадрата по углу рассеяния на отрезке $[0,2 \pi]$.

В работах [14]-[16] для $2 \lambda=-1$ длина рассеяния $a^{\prime}$ и эффективный радиус $r_{\mathrm{eff}}^{\prime}$ определялись как константы асимптотики

$$
\operatorname{ctg} \delta_{\lambda}(q) \sim \frac{2}{\pi}\left(\ln \frac{a^{\prime} q}{2}+\gamma\right)+\frac{q^{2}}{2} r_{\mathrm{eff}}^{\prime}, \quad q \rightarrow 0 .
$$

Следует отметить, что длина рассеяния $a^{\prime}$ всегда положительная, и в случае $2 \lambda=-1$ авторам работы [16] удалось получить ограничения на потенциал $V$, при которых длина рассеяния $a^{\prime}$ и эффективный радиус $r_{\text {eff }}^{\prime}$ являются конечными константами.

В том же случае $2 \lambda=-1$ в работах [17], [18] длиной рассеяния $a^{\prime \prime}$ и эффективным радиусом $r_{\text {eff }}^{\prime \prime}$ названы коэффициенты разложения

$$
\operatorname{ctg} \delta_{\lambda}(q)-\frac{2}{\pi}\left(\ln \frac{q}{2}+\gamma\right)=\frac{2}{\pi}\left(-\frac{1}{a^{\prime \prime}}+\frac{q^{2}}{2} r_{\text {eff }}^{\prime \prime}\right)+\cdots
$$

по четным степеням аргумента $q$ и доказано, что для короткодействующего потенциала такое разложение сходится равномерно на всей полуоси $q>0$. Аналогичное определение длины рассеяния $a^{\prime \prime}$ и эффективного радиуса $r_{\text {eff }}^{\prime \prime}$ использовалось в работе [19], посвященной исследованию амплитуд низкоэнергетического рассеяния в задаче трех частиц на плоскости $\mathcal{P}$.

Во всех процитированных работах [14]-[19] основное внимание уделялось случаю $2 \lambda=-1$. В работе [20] для любого $\lambda$ предложено использовать функцию эффективного радиуса

$$
K^{\prime}(q) \equiv q^{2 \lambda+1}\left[\operatorname{ctg} \delta_{\lambda}(q)-h^{\prime}(q)\right]=-\frac{1}{a^{\prime \prime \prime}}+\frac{q^{2}}{2} r_{\text {eff }}^{\prime \prime \prime}+\cdots, \quad h^{\prime}(q) \equiv \frac{2}{\pi} \ln \frac{q}{2},
$$

а константы $a^{\prime \prime \prime}$ и $r_{\text {eff }}^{\prime \prime \prime}$ считать длиной рассеяния и эффективным радиусом. Функция $h^{\prime}(q)$ была введена ранее в работе [19].

Завершим настоящий раздел определениями, введенными в работе [21] и доказанными в этой же работе утверждениями.

Для каждого состояния рассеяния $|q, \lambda\rangle, q>0$, с выбранным значением $\lambda$ функция эффективного радиуса $K(q)$ определяется через функцию $K(x ; q)$ формулами

$$
K(q) \equiv \lim _{x \rightarrow \infty} K(x ; q), \quad K(x ; q) \equiv q^{2 \lambda+1}\left[\operatorname{ctg} \delta_{\lambda}(x ; q)-h(q)\right]
$$

Функция $K(x ; q)$ является рядом по четным степеням волнового числа:

$$
K(x ; q)=\tau \sum_{n=0}^{\infty} q^{2 n} K_{n}(x)=-\frac{1}{a(x)}+\frac{q^{2}}{2} r_{\mathrm{eff}}(x)-q^{4} r_{\mathrm{eff}}^{3}(x) P(x)+\cdots, \quad x \geqslant 0 .
$$

Здесь и всюду далее $\tau-$ зависящий от значения $\lambda$ множитель,

$$
\tau \equiv \frac{2}{\pi}\left(2 \lambda-1+2 \delta_{2 \lambda,-1}\right) ! !\left(2 \lambda+1+\delta_{2 \lambda,-1}\right) ! !=\frac{\left(2^{m} m !\right)^{2}}{\pi m},
$$

где $m \equiv \lambda+1 / 2$ - целое число; $\delta_{\lambda}(x ; q)$ - фазовая функция [5], $h(q)$ - логарифмическая функция (7). 
В начальной точке $x=0$ полуоси $x \geqslant 0$ функции $a(x)$ и $\xi(x) \equiv a^{2}(x) r_{\mathrm{eff}}(x)$ равны нулю, а в бесконечно удаленной точке $x=\infty$ могут быть неограниченными. Если предельные при $x \rightarrow \infty$ значения $a$ и $r_{\text {eff }}$ функций $a(x)$ и $r_{\text {eff }}(x)$ удовлетворяют ограничениям $a \neq 0$ и $|a|<\infty,\left|r_{\text {eff }}\right|<\infty$, то вследствие представлений (9) и (10) функция $K(q)$ имеет низкоэнергетическую (при $q \rightarrow+0$ ) асимптотику

$$
K(q) \equiv q^{2 \lambda+1}\left[\operatorname{ctg} \delta_{\lambda}(q)-h(q)\right]=-\frac{1}{a}+\frac{q^{2}}{2} r_{\mathrm{eff}}-q^{4} r_{\mathrm{eff}}^{3} P+O\left(q^{6}\right),
$$

а коэффициенты $a, r_{\text {eff }}$ и $P \equiv P(\infty)$ называются длиной рассеяния, эффективным радиусом и параметром формы. Именно такое определение, исключающее особые случаи $a=0,|a|=\infty$ или $\left|r_{\text {eff }}\right|=\infty$, используется в разделах 3-5 настоящей работы. Анализу особых случаев $a=0, a= \pm \infty$ посвящен раздел 6 .

\section{3. УРАВНЕНИЯ ДЛЯ ВЫЧИСЛЕНИЯ ДЛИНЫ РАССЕЯНИЯ И ЭФФЕКТИВНОГО РАДИУСА}

В настоящем разделе ключевыми соотношениями являются разложение (10) и полученные в работе [21] специальные представления регулярного и нерегулярного решений $j_{\lambda}(q x)$ и $\tilde{n}_{\lambda}(q x)$ уравнения Шредингера $(2)$ в случае потенциала $V(x) \equiv 0$. Эти решения выражаются через функции Бесселя $J_{m}(q x)$ и $Y_{m}(q x)$ целого порядка [29] и представляют собой ряды, которые содержат известные коэффициенты $a_{n}$ и функции $d_{n}(x)$ :

$$
\begin{aligned}
& j_{\lambda}(q x)=\sqrt{\frac{\pi q x}{2}} J_{m}(q x)=\sqrt{\frac{\pi}{2}} \frac{(q x)^{\lambda+1}}{\left(2 \lambda+1+\delta_{2 \lambda,-1}\right) ! !} \sum_{n=0}^{\infty} a_{n}(q x)^{2 n}, \\
& \tilde{n}_{\lambda}(q x)=\sqrt{\frac{\pi q x}{2}} Y_{m}(q x)=n_{\lambda}(q x)+h(q) j_{\lambda}(q x) .
\end{aligned}
$$

Здесь $h(q)$ - логарифмическая функция (7) и

$$
n_{\lambda}(q x)=-\sqrt{\frac{2}{\pi}} \frac{\left(2 \lambda-1+2 \delta_{2 \lambda,-1}\right) ! !}{(q x)^{\lambda}} \sum_{n=0}^{\infty} d_{n}(x)(q x)^{2 n} .
$$

Последовательно выведем нелинейные уравнения для функций $K(x ; q), a(x)$ и линейное уравнение для функции $r_{\text {eff }}(x)$. Применим нелинейную версию метода фазовых функций [5], в которой $T(x ; q)=\operatorname{tg} \delta_{\lambda}(x ; q)$ определяется как решение уравнения

$$
\partial_{x} T(x ; q)=-\frac{\pi}{2} x V(x)\left[J_{m}(q x)-T(x ; q) Y_{m}(q x)\right]^{2}, \quad x>0,
$$

с граничным условием $T(x ; q)=0$ при $x=0$. Используя соотношения (9) и $(12)$, (13), выразим в этом уравнении функции $J_{m}$ и $Y_{m}$ через известные функции $j_{\lambda}$ и $n_{\lambda}$, а искомую функцию $T(x ; q)$ - через функцию $K(x ; q)$. В итоге получим уравнение

$$
\partial_{x} K(x ; q)=V(x)\left[q^{-\lambda-1} j_{\lambda}(\rho) K(x ; q)-q^{\lambda} n_{\lambda}(\rho)\right]^{2}, \quad x>0 .
$$

В этом нелинейном уравнении представим функции $j_{\lambda}$ и $n_{\lambda}$ как ряды (12) и (14), а искомое решение $K(x, q)$ заменим его разложением (10). В получившемся уравнении приравняем нулю сумму всех слагаемых, не зависящих от $q$, сумму всех слагаемых, пропорциональных $q^{2}$, и сумму всех слагаемых, пропорциональных $q^{4}$. Тогда 
соответственно получим нелинейное однородное уравнение для функции $a(x)$, линейное неоднородное уравнение для функции $r_{\text {eff }}(x)$ и уравнение для функции $P(x)$. Приведем уравнения для функций $a(x)$ и $r_{\mathrm{eff}}(x)$. Для краткости записи ниже мы используем обозначение $y(x) \equiv \ln x$ и не указываем аргумент $x$ функций $y(x), V(x)$, $a(x)$ и $r_{\text {eff }}(x)$.

В случае $2 \lambda=-1$ функция $a(x)$ подчиняется уравнению

$$
\tau \partial_{x} a=x V[1+\tau a y]^{2}
$$

а в случае $2 \lambda \geqslant 1-$ уравнению

$$
\tau \partial_{x} a=\frac{x^{2 \lambda}}{2 \lambda+1} V\left[x-x^{-2 \lambda} \tau a\right]^{2} .
$$

В случае $2 \lambda=-1$ функция $r_{\text {eff }}(x)$ удовлетворяет уравнению

$$
\tau \partial_{x} r_{\mathrm{eff}}=-a^{-2} x V[1+\tau a y]\left[2 a r_{\mathrm{eff}}+x^{2}(1+\tau a y-\tau a)\right]
$$

в случае $2 \lambda=1-$ уравнению

$$
\tau \partial_{x} r_{\mathrm{eff}}=a^{-2} x V\left[\tau a-x^{2}\right]\left(a\left(r_{\mathrm{eff}}-\tau y\right)+\frac{x^{2}}{4}\right)
$$

и, наконец, в оставшемся случае $2 \lambda \geqslant 3-$ уравнению

$$
\tau \partial_{x} r_{\mathrm{eff}}=\frac{2}{2 \lambda+1} a^{-2} x V\left(\tau a-x^{2 \lambda+1}\right)\left[a\left(r_{\mathrm{eff}}+\tau \frac{x^{-2 \lambda+1}}{2 \lambda-1}\right)+\frac{x^{2}}{2 \lambda+3}\right] .
$$

Обсудим уравнения (17) и (18). В этих уравнениях знаки производной $\partial_{x} a$ и потенциала $V$ совпадают, эта производная равна нулю в тех же точках, что и потенциал. Следовательно, непрерывное решение $a(x)$ монотонно возрастает или убывает на отрезке $\left[x_{1}, x_{2}\right]$, если на этом отрезке потенциал является соответственно отталкивающим $(V>0)$ или притягивающим $(V<0)$. Решение имеет локальный экстремум в некоторой точке $x=\tilde{x}<\infty$, если в этой точке потенциал меняет знак. Уравнения (17) могут иметь решения, терпящие разрыв второго рода. В этом случае в малой окрестности точки разрыва следует перейти к уравнениям для функции $\tilde{a}(x)=1 / a(x)$.

Теперь обсудим уравнения (19)-(21). Их правые части содержат множитель $a^{-2}(x)$, и поэтому решение $r_{\mathrm{eff}}(x)$ может иметь полюс первого или второго порядка в некоторой точке $x=\tilde{x}$, если в этой точке функция $a(x)$ меняет знак. При наличии полюса следует положить $r_{\mathrm{eff}}(x)=\tau \xi(x) / a^{2}(x)$ и вместо исходных уравнений использовать порожденные ими уравнения для ограниченной в точке $x=\tilde{x}$ функции $\xi(x)$ : в случае $2 \lambda=-1$ мы имеем уравнение

$$
\partial_{x} \xi=\frac{x}{2} V[1+\tau a y]\left[4 y \xi-x^{2}(1+\tau a y-\tau a)\right],
$$

при $2 \lambda=1-$ уравнение

$$
\partial_{x} \xi=\frac{1}{8 x} V\left[\tau a-x^{2}\right]\left[8 \xi+x^{2}\left(x^{2}-4 \tau a y\right)\right],
$$


а в случае $2 \lambda \geqslant 3$ - уравнение

$$
\partial_{x} \xi=\frac{1}{2 \lambda+1} V\left[\tau a-x^{2 \lambda+1}\right] x^{-2 \lambda}\left(2 \xi+\frac{\tau a x^{2}}{2 \lambda-1}+\frac{x^{2 \lambda+3}}{2 \lambda+3}\right) .
$$

Заметим, что равенства $a(0)=0$ и $\xi(0)=0$, упомянутые в предыдущем разделе, являются граничными условиями для уравнений $(17),(18)$ и $(22)-(24)$ в начальной точке $x=0$, каждое из трех уравнений (22)-(24) представимо в виде

$$
\partial_{x} \xi(x)=v_{2}(x) \xi(x)+v_{1}(x),
$$

и поэтому при условии $\xi(0)=0$ оно имеет единственное решение

$$
\xi(x)=\int_{0}^{x} d x_{1} v_{1}\left(x_{1}\right) \exp \left\{\int_{x_{1}}^{x} d x_{2} v_{2}\left(x_{2}\right)\right\},
$$

которое является интегральным представлением функции $\xi(x)$ через функцию $a(x)$.

Наша следующая задача - свести все полученные уравнения (17)-(21) к системам линейных дифференциальных уравнений первого порядка. Используем для этого известный в теории дифференциальных уравнений [27] метод вариации произвольных коэффициентов. Напомним, что в этом методе искомое решение исходного уравнения сначала представляется через две неизвестные функции, производные которых по определению подчиняются некоторому тождеству, а затем это тождество используется при выводе системы двух уравнений.

Начнем с линеаризации уравнения (17). Его решение будем искать в виде

$$
a(x)=-\frac{s_{0}(x)}{\tau c_{0}(x)} .
$$

Произвольные функции $c_{0}(x)$ и $s_{0}(x)$ подчиним тождеству

$$
\partial_{x} c_{0}(x) \equiv \ln x \partial_{x} s_{0}(x), \quad x>0 .
$$

Подстановкой формулы (26) сведем исходное уравнение (17) к соотношению, содержащему обе производные $\partial_{x} c_{0}$ и $\partial_{x} s_{0}$. Используя тождество $(27)$, исключим из этого соотношения производную $\partial_{x} s_{0}$ или производную $\partial_{x} c_{0}$. Получим два уравнения для искомых функций $c_{0}(x)$ и $s_{0}(x)$. Запишем полученную таким образом однородную систему двух уравнений в следующем виде:

$$
\begin{aligned}
& \partial_{x} c_{0}(x)=-V(x)\left[c_{0}(x)-s_{0}(x) \ln x\right] x \ln x, \quad 2 \lambda=-1 . \\
& \partial_{x} s_{0}(x)=-V(x)\left[c_{0}(x)-s_{0}(x) \ln x\right] x,
\end{aligned}
$$

Теперь изложенным выше способом линеаризуем уравнение (18). Для этого используем подстановку (26), а искомые функции $c_{0}$ и $s_{0}(x)$ подчиним тождеству

$$
\partial_{x} c_{0}(x) \equiv-x^{-2 \lambda-1} \partial_{x} s_{0}(x), \quad x>0, \quad 2 \lambda \geqslant 1 .
$$

В результате получим однородную систему линейных уравнений

$$
\begin{aligned}
& \partial_{x} c_{0}(x)=\frac{V(x)}{2 \lambda+1}\left[x c_{0}(x)+x^{-2 \lambda} s_{0}(x)\right], \\
& \partial_{x} s_{0}(x)=-\frac{V(x)}{2 \lambda+1}\left[x c_{0}(x)+x^{-2 \lambda} s_{0}(x)\right] x^{2 \lambda+1},
\end{aligned}
$$


Для редукции уравнений (19)-(21) к системам неоднородных уравнений применим подстановку

$$
r_{\mathrm{eff}}(x)=\frac{2 \tau}{s_{0}(x)}\left(c_{1}(x)-s_{1}(x) \frac{c_{0}(x)}{s_{0}(x)}\right)
$$

и используем соответствующие выбранному значению $\lambda, 2 \lambda \geqslant-1$, уравнения (28) или (30) и различные тождества. Далее мы приведем эти тождества и итоговые системы уравнений, полагая для краткости записи $y(x) \equiv \ln x$ и не указывая аргумент $x$ функций $y(x), V(x), c_{0,1}(x)$ и $s_{0,1}(x)$.

Итак, уравнение (19) благодаря тождеству

$$
4\left(\partial_{x} c_{1}-y \partial_{x} s_{1}\right) \equiv x^{2} \partial_{x} s_{0}, \quad x>0,
$$

сводится к системе уравнений

$$
\begin{aligned}
\partial_{x} c_{1} & =\frac{V}{4}\left\{4 y\left(c_{1}-y s_{1}\right)+x^{2}\left[(2 y-1) c_{0}+2(1-y) y s_{0}\right]\right\} x, \quad 2 \lambda=-1 . \\
\partial_{x} s_{1} & =\frac{V}{4}\left\{4\left(y s_{1}-c_{1}\right)+x^{2}\left[2 c_{0}+(1-2 y) s_{0}\right]\right\} x
\end{aligned}
$$

Тождество

$$
8\left(\partial_{x} c_{1}+x^{-2} \partial_{x} s_{1}\right) \equiv x^{2} \partial_{x} c_{0}+4 y \partial_{x} s_{0}, \quad x>0,
$$

позволяет вывести из уравнения (20) систему уравнений

$$
\begin{aligned}
& \partial_{x} c_{1}=\frac{V}{16}\left[8\left(x c_{1}+\frac{1}{x} s_{1}\right)-x^{3}(4 y+1) c_{0}-8 x y s_{0}\right], \\
& \partial_{x} s_{1}=-\frac{V}{16}\left[8\left(x c_{1}+\frac{1}{x} s_{1}\right)-2 x^{3} c_{0}-x(4 y+1) s_{0}\right] x^{2},
\end{aligned}
$$

При использовании тождества

$$
2\left(\partial_{x} c_{1}+x^{-2 \lambda-1} \partial_{x} s_{1}\right) \equiv \frac{x^{2}}{2 \lambda+3} \partial_{x} c_{0}-\frac{x^{-2 \lambda-1}}{2 \lambda-1} \partial_{x} s_{0}, \quad x>0, \quad 2 \lambda \geqslant 3,
$$

уравнение $(21)$ порождает следующую систему в случае $2 \lambda \geqslant 3$ :

$$
\begin{aligned}
& \partial_{x} c_{1}=\frac{V}{2 \lambda+1}\left[x c_{1}+x^{-2 \lambda} s_{1}+\frac{x^{2}}{2 \lambda-1}\left(\frac{2}{2 \lambda+3} x c_{0}+x^{-2 \lambda} s_{0}\right)\right] \\
& \partial_{r} s_{1}=-\frac{V}{2 \lambda+1}\left[x c_{1}+x^{-2 \lambda} s_{1}-\frac{x^{2}}{2 \lambda+3}\left(x c_{0}-\frac{2}{2 \lambda-1} x^{-2 \lambda} s_{0}\right)\right] x^{2 \lambda+1} .
\end{aligned}
$$

Завершим настоящий раздел важными замечаниями.

Функции $K(x ; q), a(x)$ и $r_{\text {eff }}(x)$ имеют прозрачный физический смысл: их значения в любой точке $x=b \geqslant 0$ суть соответственно значения функции эффективного радиуса, длины рассеяния и эффективного радиуса, если потенциал $V(x)$ заменить потенциалом $V(x) \theta(x-b)$, т. е. "обрезать" в этой точке. Правые части уравнений (19)-(21) для функции $r_{\text {eff }}(x)$ содержат функцию $a(x)$, а связь $r_{\text {eff }}(x)=$ $\tau \xi(x) / a^{2}(x)$ и соотношение (25) порождают интегральное представление функции $r_{\text {eff }}(x)$ через функцию $a(x)$. Следовательно, эффективный радиус $r_{\text {eff }}$ зависит и от 
потенциала $V(x)$, и от длины рассеяния $a$. Анализ функции $P(x)$ выходит за рамки настоящей работы, поэтому уравнение, определяющее эту функцию, лишь упоминалось выше.

В отличие от функций $a(x)$ и $r_{\text {eff }}(x)$ компоненты $c_{n}(x)$ и $s_{n}(x), n=1,2$, не имеют какого-либо физического смысла. Системы уравнений (28), (30) и (33), (35), (37) принадлежат бесконечной по индексу $n=0,1, \ldots$ рекуррентной цепочке уравнений, которая получена в работе [21] способом, отличным от изложенного выше. В этой же работе доказаны следующие утверждения. Условие (3) порождает граничные условия $c_{n}(x)=\delta_{n, 0}, s_{n}(x)=0, n=0,1, \ldots$, в начальной точке $x=0$. При таких условиях и ограничениях $(1)$ на потенциал $V(x)$ обсуждаемая цепочка уравнений имеет единственное решение, все функции $c_{n}(x)$ и $s_{n}(x), n=0,1, \ldots$, непрерывны на полуоси $x>0$ и ограничены в бесконечно удаленной точке $x=\infty$. Вследствие этих фактов и связей (26) и (31) при тех же ограничениях (1) уравнения (17)-(21) однозначно разрешимы.

Обсудим преимущества и недостатки исходных нелинейных уравнений (17)-(21) и выведенных из них систем линейных уравнений (28), (30), (33), (35) и (37).

Первое и неоспоримое преимущество нелинейных уравнений (17)-(21) состоит в том, что их решения $a(x)$ и $r_{\text {eff }}(x)$ обладают прозрачным физическим смыслом. Второе преимущество этих уравнений заключается в том, что, используя их, можно вычислить длину рассеяния $a$ и эффективный радиус $r_{\text {eff }}$ наиболее экономичным способом: для этого достаточно численно проинтегрировать одно уравнение (17) или (18), а затем вычислить один интеграл (25). Как пояснялось выше, решение $a(x)$ нелинейных уравнений (17) или (18) может быть неограниченным в некоторой точке $x=t>0$, но этот единственный недостаток нетрудно преодолеть, перейдя при вычислении функции $a(x)$ в малой окрестности точки $x=t$ к уравнению для функции $1 / a(x)$.

Единственное преимущество систем линейных уравнений (28), (30), (33), (35) и (37) по сравнению с исходными нелинейными уравнениями (17)-(21) таково: все компоненты $c_{n}(x)$ и $s_{n}(x)$ решений $\left\{c_{n}(x), s_{n}(x)\right\}, n=0,1$, этих систем являются всюду ограниченными функциями. Однако эти функции не имеют никакого физического смысла, а вычисление длины рассеяния $a$ и эффективного радиуса $r_{\text {eff }}$ требует численного интегрирования четырех зацепляющихся уравнений. Например, в случае $2 \lambda=-1$ придется одновременно интегрировать систему (28) и систему (33). Ясно, что такой способ вычисления длины рассеяния и эффективного радиуса менее экономичен, чем упомянутый выше алгоритм, основанный на нелинейных уравнениях (17) или (18) и интегральном представлении (25) эффективного радиуса $r_{\text {eff }}$ через функцию $a(x)$.

\section{4. ДЛИНА РАССЕЯНИЯ И ЭФФЕКТИВНЫЙ РАДИУС В СЛУЧАЕ ПОТЕНЦИАЛА ПРЯМОУГОЛЬНОЙ ФОРМЫ}

Приведем полезный с методической точки зрения пример, позволяющий проиллюстрировать все возможные особенности функций $a(x)$ и $r_{\mathrm{eff}}(x)$. Для этого исследуем случай финитного потенциала прямоугольной формы. Положим $V(x)=$ $V_{0} \theta(b-x)$, где $V_{0} \neq 0$ и $b>0$ - некоторые константы. Наша задача - найти и исследовать точные решения уравнений (17)-(21) на всей полуоси $x>0$. Так как 
в области $x>b$ потенциал $V(x)$ тождественно равен нулю, в этой области искомые решения $a(x)$ и $r_{\mathrm{eff}}(x)$ тождественно равны их значениям $a(b)$ и $r_{\mathrm{eff}}(b)$ в точке $x=b$. Следовательно, эти значения являются длиной рассеяния $a$ и эффективным радиусом $r_{\text {eff }}$, и для вычисления этих констант достаточно решить уравнения (17)-(21) на конечном отрезке $0 \leqslant x \leqslant b$. Приступим к решению.

Сначала рассмотрим случай $V_{0}<0$, когда потенциал $V(x)$ является прямоугольной ямой, глубина и ширина которой равны соответственно $-V_{0}$ и $b$. Положив $\alpha \equiv \sqrt{-V_{0}}, z \equiv \alpha x$ и выполнив соответствующую подстановку

$$
a(x)=-\frac{1}{\tau} x^{2 m} \frac{z \partial_{z} A(z)+m A(z)}{z \partial_{z} A(z)\left[\delta_{m, 0} \ln x+\theta(m-1)\right]-\left(\delta_{m, 0}+m\right) A(z)},
$$

сведем уравнения (17) и (18) к уравнению Бесселя [29] для функции $A(z)$. Решив такие уравнения, выведем представление функции $a(x)$ через функции Бесселя $J_{m}(z)$ и найдем интеграл $(25)$, а затем и соответствующую ему функцию $r_{\mathrm{eff}}(x)$. В итоге получим следующие формулы: для $x \leqslant b$ в случае $2 \lambda=-1$

$$
a(x)=-\frac{\pi}{2} \frac{z J_{1}(z)}{J_{0}(z)+z J_{1}(z) \ln x}, \quad r_{\mathrm{eff}}(x)=\frac{1}{\pi \alpha^{2}}\left(z^{2}-2+2 z \frac{J_{0}(z)}{J_{1}(z)}\right) ;
$$

в случае $2 \lambda=1$

$$
a(x)=-\frac{\pi}{4} x^{2} \frac{J_{2}(z)}{J_{0}(z)}, \quad r_{\mathrm{eff}}(x)=\frac{1}{\pi}\left(4 \ln x+\left[8 J_{2}(z)-z^{2} J_{0}(z)\right] \frac{J_{0}(z)}{\left[z J_{2}(z)\right]^{2}}\right) ;
$$

если $2 \lambda \geqslant 3$, то

$$
\begin{aligned}
a(x) & =-\pi m\left(\frac{x^{m}}{2^{m} m !}\right)^{2} B_{m}^{-1}(z), \\
r_{\mathrm{eff}}(x) & =-\frac{\left(2^{m} m !\right)^{2}}{2 \pi m}\left(\frac{\alpha^{m-1}}{z^{m}}\right)^{2}\left[z^{2}\left(\frac{1}{m-1}+\frac{B_{m}^{2}(z)}{m+1}\right)-4 m B_{m}(z)\right],
\end{aligned}
$$

где

$$
B_{m}(z) \equiv \frac{J_{m-1}(z)}{J_{m+1}(z)} .
$$

Теперь несложно рассмотреть случай $V_{0}>0$, когда потенциал $V(x)=V_{0} \theta(b-x)$ является прямоугольным барьером, высота и ширина которого равны соответственно $V_{0}$ и $b$. Выполнив в формулах (38)-(40) замены

$$
\alpha \mapsto i \alpha=i \sqrt{V_{0}}, \quad z \mapsto i z=i \alpha x, \quad J_{m}(i z) \mapsto i^{m} I_{m}(z),
$$

получим следующие представления функций $a(x)$ и $r_{\mathrm{eff}}(x)$ через модифицированные функции Бесселя $I_{m}(z)$ : для $x \leqslant b$ в случае $2 \lambda=-1$

$$
a(x)=\frac{\pi}{2} \frac{z I_{1}(z)}{I_{0}(z)-z I_{1}(z) \ln x}, \quad r_{\mathrm{eff}}(x)=\frac{1}{\pi \alpha^{2}}\left(z^{2}+2-2 z \frac{I_{0}(z)}{I_{1}(z)}\right) ;
$$

в случае $2 \lambda=1$

$$
a(x)=\frac{\pi}{4} x^{2} \frac{I_{2}(z)}{I_{0}(z)}, \quad r_{\mathrm{eff}}(x)=\frac{1}{\pi}\left(4 \ln x+\left[8 I_{2}(z)-z^{2} I_{0}(z)\right] \frac{I_{0}(z)}{\left[z I_{2}(z)\right]^{2}}\right) ;
$$


если $2 \lambda \geqslant 3$, то

$$
\begin{aligned}
a(x) & =\pi m\left(\frac{x^{m}}{2^{m} m !}\right)^{2} B_{m}^{-1}(z), \\
r_{\mathrm{eff}}(x) & =-\frac{\left(2^{m} m !\right)^{2}}{2 \pi m}\left(\frac{\alpha^{m-1}}{z^{m}}\right)^{2}\left\{z^{2}\left(\frac{1}{m-1}+\frac{B_{m}^{2}(z)}{m+1}\right)-4 m B_{m}(z)\right\},
\end{aligned}
$$

где теперь

$$
B_{m}(z) \equiv \frac{I_{m-1}(z)}{I_{m+1}(z)} .
$$

Обсудим особенности поведения функций (38)-(40) на отрезке $0 \leqslant x \leqslant b$.

Как известно [29], в области $z>0$ все нули $\gamma_{m+1, i}, i=1,2, \ldots$, функции $J_{m+1}(z)$ являются простыми. Поэтому при любом значении $\lambda$ на полуинтервале $0<x \leqslant b$ функция $a(x)$ или не имеет нулей, если $\alpha b<\gamma_{m+1,1}, m=\lambda+1 / 2$, или имеет конечное число $n$ простых нулей $x_{m+1, j}^{+}=\gamma_{m+1, j} / \alpha, j=1,2, \ldots, n$. Число $n$ равно максимальному значению номера $i$, при котором выполняется условие $\gamma_{m+1, i} \leqslant \alpha b$. При любом $\lambda$ нули $x_{m+1, j}^{+}$функции $a(x)$ являются полюсами соответствующей функции $r_{\text {eff }}(x)$. В случае $2 \lambda=-1$ эти полюсы имеют первый порядок, а при условии $2 \lambda \geqslant 1-$ второй.

Случай $2 \lambda=-1$ является особым. В этом случае согласно формулам (38) все полюсы $x_{1, j}^{-}$функции $a(x)$ связаны с нулями $z_{j}$ функции $J_{0}(z)+z J_{1}(z) \ln x$ соотношениями $x_{1, j}^{-}=z_{j} / \alpha$. Все нули $z_{j}$ простые. Поэтому на отрезке $0 \leqslant x \leqslant b$ функция $a(x)$ является гладкой, если $z_{1}>\alpha b$, а в противном случае имеет конечное число $n$ полюсов $x_{1, j}^{-}=z_{j} / \alpha, j=1,2, \ldots, n$, первого порядка. Функция $r_{\text {eff }}(x)$ в точке $x=0$ ограничена и отлична от нуля.

При любом $\lambda \geqslant 1 / 2$ в силу равенств $(39),(40)$ функция $a(x)$ непрерывна на отрезке $0 \leqslant x \leqslant b$, если $\gamma_{m-1,1}(z)>\alpha b$, а при условии $\gamma_{m-1, n} \leqslant b$ имеет конечное число $n$ полюсов первого порядка $x_{m-1, j}=\gamma_{m-1, j}, j=1,2, \ldots, n$. В случае $2 \lambda \geqslant 1$ точка $x=0$ для функции $r_{\text {eff }}(x)$ является полюсом второго порядка.

Так как нули $\gamma_{m-1, j}$ и $\gamma_{m+1, j}$ функции $J_{m-1}(z)$ и $J_{m+1}(z)$ перемежаются [29], при любом $\lambda$ этим же свойством обладают полюсы $x_{m, j}^{-}$и нули $x_{m, j}^{+}$функции $a(x)$ :

$$
x_{m, j}^{-}<x_{m, j}^{+}<x_{m, j+1}^{-}<x_{m, j+1}^{+}, \quad j=1,2, \ldots, n-1 .
$$

По той же причине при любом $\lambda$ перемежаются нули и полюсы функции $r_{\mathrm{eff}}(x)$.

Теперь перечислим основные особенности функций (42)-(44).

Известно [29], что на полуоси $z>0$ все функции $I_{m}(z)$ положительные и монотонно возрастающие. Поэтому при любом $\lambda>1 / 2$ согласно формулам (43), (44) такими же свойствами обладает функция $a(x)$, а функция $r_{\mathrm{eff}}(x)$ монотонно возрастает, но может иметь только один, причем простой, нуль, принадлежащий отрезку $0 \leqslant x \leqslant b$, и только один полюс $x=0$, причем второго порядка.

Случай $2 \lambda=-1$, когда функции $a(x)$ и $r_{\text {eff }}(x)$ определены формулами $(42)$, является исключительным. В этом случае на полуоси $z>0$ функция $I_{0}(z)-z I_{1}(z) \ln x$ имеет один простой нуль $z_{1}$. Поэтому на отрезке $0 \leqslant x \leqslant b$ функция $a(x)$ является положительной и монотонно возрастающей, если $z_{1}<\alpha b$, а в противном случае эта 


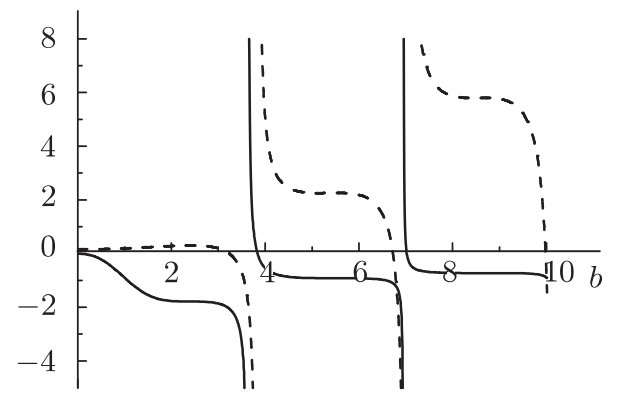

a

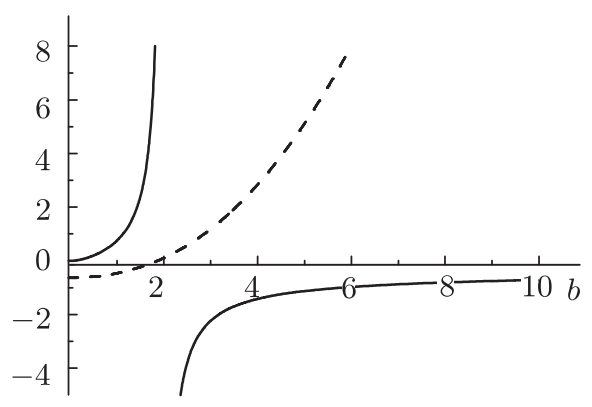

B

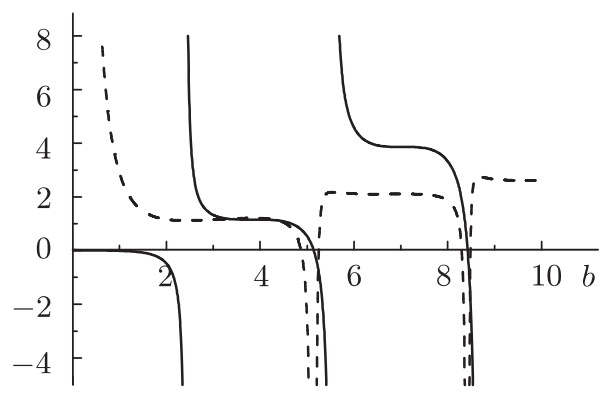

6

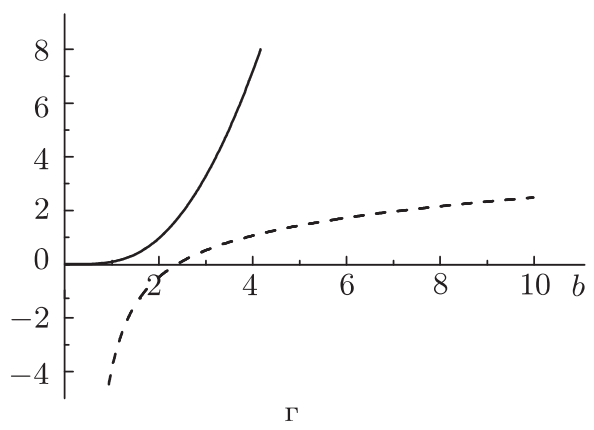

Рис. 1. Зависимость масштабированных длины рассеяния $\tau_{1} a(b)$ (сплошная кривая) и эффективного радиуса $\tau_{2} r_{\mathrm{eff}}(b)$ (штриховая кривая) от ширины $b$ потенциала $V(x)=V_{0} \theta(b-x)$ прямоугольной формы в четырех случаях: при $V_{0}=-1,2 \lambda=-1, \tau_{1}=1, \tau_{2}=1 / 4(\mathrm{a})$, при $V_{0}=-1,2 \lambda=1, \tau_{1}=1 / 10$, $\tau_{2}=1$ (б), при $V_{0}=1,2 \lambda=-1, \tau_{1}=1, \tau_{2}=1$ (в) и при $V_{0}=1,2 \lambda=1, \tau_{1}=1$, $\tau_{2}=1(г)$.

функция имеет полюс первого порядка в точке $x_{1,1}^{-}=z_{1} / \alpha$. Функция $r_{\text {eff }}(x)$ ограничена в точке $x=0$ и монотонно возрастает в области $0<x \leqslant b$, в этой области она может иметь только один нуль, причем первого порядка.

Все перечисленные выше особенности функций $a(x)$ и $r_{\text {eff }}(x)$, а также зависимость длины рассеяния $a(b)$ и эффективного радиуса $r_{\text {eff }}(b)$ от ширины $b$ потенциала $V=V_{0} \theta(b-x)$ прямоугольной формы в четыре случаях $V_{0}= \pm 1,2 \lambda= \pm 1$ иллюстрирует рис. 1. Видно, что в этих случаях длина рассеяния и эффективный радиус могут принимать любые вещественные значения, кроме того, имеет место описанная в предыдущем разделе корреляция между бесконечно малыми и бесконечно большими по абсолютной величине значениями этих параметров.

Как показано в работе [21], если известны функции $c_{n}(x)$ и $s_{n}(x)$, удовлетворяющие системам (28), (30), (33), (35), (37) и граничным условиям $c_{n}(0)=\delta_{n, 0}$ и $s_{n}(0)=0, n=0,1$, то нетрудно построить явную низкоэнергетическую асимптотику решения $u_{\lambda}(x ; q)$ краевой задачи Шредингера $(2)-(4)$. Поэтому имеет смысл найти точные решения этих систем в случае потенциала прямоугольной формы.

Приступим к решению такой задачи. Сначала положим $V(x)=V_{0} \theta(b-x), V_{0}<0$, $\alpha=\sqrt{-V_{0}}$. Исследуем систему двух уравнений (28). В отличие от первого уравне- 
ния ее второе уравнение не содержит квадрата логарифмической функции и поэтому является более простым. Продифференцируем это уравнение по аргументу $x$. Используя тождество $(27)$, исключим из полученного уравнения производную $\partial_{x} c_{0}(x)$. В результате выведем однородное уравнение

$$
\left(\partial_{x}^{2}-x^{-1} \partial_{x}+\alpha^{2}\right) s_{0}(x)=0, \quad x>0,
$$

с граничным условием $s_{0}(0)=0$. Подстановкой $z=\alpha x, s_{0}(x)=z Z(z)$ сведем это уравнение к известному уравнению [29] для функции Бесселя $J_{1}(z)$ первого порядка. Теперь подставим найденную компоненту $s_{0}(x)=z J_{1}(z)$ в тождество (27). Полученное таким образом представление производной $\partial_{x} c_{0}(x)$ запишем на отрезке $[0, x]$ в интегральной форме. Для этого используем граничное условие $c_{0}(0)=1$. Взяв интеграл, получим, что $c_{0}(x)=J_{0}(z)+z J_{1}(z) \ln x$.

Аналогичным способом последовательно исследуем оставшиеся системы уравнений (30), (33), (35) и (37). Решение каждой из них начнем с дифференцирования ее второго уравнения, не содержащего в отличие от первого уравнения степенного множителя $x^{2 \lambda+1}$. Для исключения производных $\partial_{x} s_{0}(x)$ или $\partial_{x} s_{1}(x)$ из искомого уравнения для компонент $c_{0}(x)$ или $c_{1}(x)$ используем соответствующие выбранному значению $\lambda$ тождества (29), (32), (34) или (36). После того как получено явное решение уравнения для компоненты $c_{0}(x)$ или $c_{1}(x)$, найдем производную этой компоненты по аргументу $x$. Подставив эту производную в соответствующее тождество, получим представление производной $\partial_{x} s_{0}(x)$ или $\partial_{x} s_{1}(x)$. Используя граничное условие $s_{0,1}(0)=0$, запишем это представление в виде интеграла. Вычислив его, найдем компоненту $s_{0}(x)$ или $s_{1}(x)$.

Приведем найденные описанным выше способом представления компонент $c_{0,1}(x)$ и $s_{0,1}(x)$ через функции Бесселя $J_{m}(z)$ аргумента $z=\alpha x$, где $\alpha \equiv \sqrt{-V_{0}}, V_{0}<0$.

Итак, для потенциала $V(x)$ в виде прямоугольной ямы $V(x)=V_{0} \theta(b-x), V_{0}<0$, при $x \leqslant b$ имеем следующие решения.

В случае $2 \lambda=-1$

$$
\begin{aligned}
c_{0}(x) & =J_{0}(z)+z J_{1}(z) \ln x, & s_{0}(x) & =z J_{1}(z), \\
c_{1}(x) & =\frac{x^{2}}{4}\left[z J_{1}(z)(1-\ln x)-J_{2}(z)\right], & s_{1}(x) & =-\alpha \frac{x^{3}}{4} J_{1}(z) .
\end{aligned}
$$

В случае $2 \lambda=1$

$$
\begin{aligned}
c_{0}(x) & =J_{0}(z), & s_{0}(x) & =x^{2} J_{2}(z), \\
c_{1}(x) & =\frac{x^{2}}{2} J_{2}(z) \ln x, & s_{1}(x) & =\frac{x^{2}}{8 \alpha^{2}}\left[z^{2} J_{0}(z)-8 J_{2}(z)\right] .
\end{aligned}
$$

В случае $2 \lambda \geqslant 3$

$$
\begin{array}{rlrl}
c_{0}(x)=w z^{1-m} J_{m-1}(z), & s_{0}(x) & =w \alpha^{-2 m} z^{m+1} J_{m+1}(z), \\
c_{1}(x)=-\frac{w}{4(m-1) \alpha^{2}} z^{3-m} J_{m+1}(z), & s_{1}(x) & =\frac{w}{\alpha^{2 m+2}} \times \\
& \times\left(\frac{z^{2} J_{m-1}(z)}{4(m+1)}-m J_{m+1}(z)\right),
\end{array}
$$

где $w \equiv 2^{m-1}(m-1) !$. 
На полуоси $x>b$ каждая из компонент $c_{0,1}(x)$ и $s_{0,1}(x)$ тождественно равна своему значению в точке $x=b$.

Теперь нетрудно найти компоненты $c_{0,1}(x), s_{0,1}(x)$ для потенциала $V(x)$ в виде прямоугольного барьера: $V(x)=V_{0} \theta(b-x), V_{0}>0$. Выполнив замены (41) в формулах (45)-(47), получим следующие представления этих компонент при $x \leqslant b$ через модифицированные функции Бесселя $I_{m}(z)$, где $z=\alpha x, \alpha=\sqrt{V_{0}}$.

В случае $2 \lambda=-1$

$$
\begin{array}{ll}
c_{0}(x)=I_{0}(z)-z I_{1}(z) \ln x, & s_{0}(x)=-z I_{1}(z), \\
c_{1}(x)=-\frac{x^{2}}{4}\left[z I_{1}(z)(1-\ln x)-I_{2}(z)\right], & s_{1}(x)=\alpha \frac{x^{3}}{4} I_{1}(z) .
\end{array}
$$

В случае $2 \lambda=1$

$$
\begin{aligned}
c_{0}(x) & =I_{0}(z), & s_{0}(x) & =-x^{2} I_{2}(z), \\
c_{1}(x) & =-\frac{x^{2}}{2} I_{2}(z) \ln x, & s_{1}(x) & =\frac{x^{2}}{8 \alpha^{2}}\left[z^{2} I_{0}(z)-8 I_{2}(z)\right] .
\end{aligned}
$$

Наконец, в случае $2 \lambda \geqslant 3$

$$
\begin{array}{rlrl}
c_{0}(x)=w z^{1-m} I_{m-1}(z), & s_{0}(x) & =-w \alpha^{-2 m} z^{m+1} I_{m+1}(z), \\
c_{1}(x)=\frac{w}{4(m-1) \alpha^{2}} z^{3-m} I_{m+1}(z), & s_{1}(x) & =\frac{w}{\alpha^{2 m+2}} \times \\
& \times\left(\frac{z^{2} I_{m-1}(z)}{4(m+1)}-m I_{m+1}(z)\right),
\end{array}
$$

где, как и выше, $w \equiv 2^{m-1}(m-1)$ !.

В области $x>b$ все компоненты $c_{0,1}(x)$ и $s_{0,1}(x)$ тождественно равны своим значениям в точке $x=b$.

\section{5. ДЛИНА И ЭФФЕКТИВНЫЙ РАДИУС ДВУМЕРНОГО РАССЕЯНИЯ НЕЙТРОНА НА ПРОТОНЕ}

Параметры низкоэнергетического рассеяния $a$ и $r_{\text {eff }}$ нейтрона $n$ на протоне $p$ в двумерной плоскости, по-видимому, ранее не вычислялись. Восполним этот недостаток современной ядерной физики и попутно сравним все известные методы вычисления констант $a$ и $r_{\text {eff. }}$.

Предположим, что полное взаимодействие между нейтроном и протоном в их синглетном по полному спину состоянии зависит только от расстояния $r$ между ними и исчерпывается ${ }^{1} S_{0}$-потенциалом Рида $V^{\mathrm{s}}(r)$ с мягким кором [6]:

$$
V^{\mathrm{s}}(r)=\frac{g(z)}{z}\left\{V_{1}+g^{3}(z)\left[V_{4}+V_{7} g^{3}(z)\right]\right\}, \quad g(z) \equiv e^{-z}, \quad z \equiv \frac{r}{r_{\pi}},
$$

где

$$
V_{1}=-10.463 \mathrm{M \ni B}, \quad V_{4}=-1650.6 \mathrm{M \ni B}, \quad V_{7}=6484.2 \mathrm{M \ni B}, \quad r_{\pi} \equiv \frac{10}{7} \Phi_{\mathrm{M}} .
$$

Наглядное представление о поведении потенциала $V^{\mathrm{s}}(r)$ дает рис. 2. С ростом аргумента $r$ потенциал $V^{\mathrm{s}}(r)$ монотонно убывает на интервале $\left(0, r_{2}^{\mathrm{s}}\right)$, обращается в нуль 


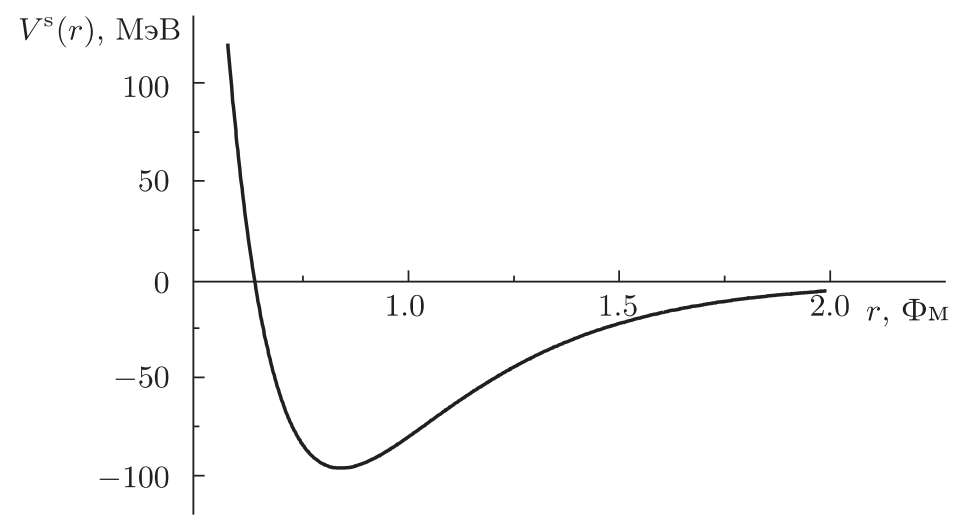

Рис. 2. Потенциал $V^{\mathrm{s}}(r)$, заданный формулами (51).

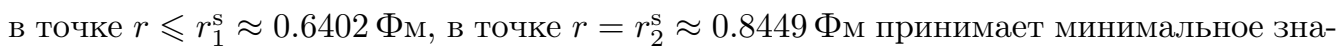
чение $V^{\mathrm{s}}\left(r_{2}^{\mathrm{s}}\right)=V_{\min }^{\mathrm{s}} \approx-97.2308 \mathrm{MэB}$, а при $r>r_{1}^{\mathrm{s}}$ монотонно возрастает, стремясь к нулю при $r / r_{\pi} \rightarrow \infty$. Обсуждаемый потенциал имеет асимптотики

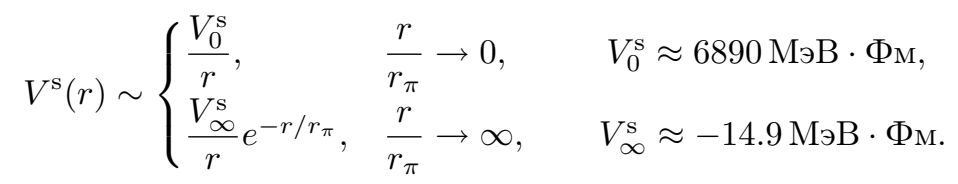

В области $r \leqslant r_{1}^{\mathrm{s}} \approx 0.6402 \Phi_{\mathrm{M}}$ данный потенциал является отталкивающим $\left(V^{\mathrm{s}}>0\right)$, а в области $r>r_{1}^{\mathrm{s}}-$ притягивающим $\left(V^{\mathrm{s}}<0\right)$.

Константу $\hbar^{2} / 2 \mu$, где $\mu$ - приведенная масса $n p$-системы, положим равной ее известному значению [6] $41.47 \mathrm{MэB} \cdot \Phi_{\mathrm{M}^{2}}$. Безразмерный аргумент $x$ определим соотношением $x=r / d$. В качестве $d$ выберем характерную для ядерных систем длину $d=1 \Phi_{\mathrm{M}}$.

Используем систему центра масс нейтрона и протона. В этой системе задача двумерного $n p$-рассеяния сводится к движению квантовой частицы $p_{1}$ с массой $m_{1}$, равной приведенной массе $n p$-системы, в поле короткодействующего потенциала $V(x)=g_{\mathrm{s}} V^{\mathrm{s}}(r)$, где $x=r / d$, a $g_{\mathrm{s}} \equiv 2 m_{1}(d / \hbar)^{2}$.

Поясним схему наших вычислений, выполненных с относительной точностью до десяти знаков. Функция $a(x)$ вычислялась путем численного интегрирования нелинейного уравнения (17) или (18), а функция $r_{\text {eff }}(x)$ рассчитывалась как соответствующий уже найденной функции $a(x)$ однократный интеграл $(25)$. Длина рассеяния $a$ и эффективный радиус $r_{\text {eff }}$ определялись как значения функций $a(x)$ и $r_{\text {eff }}(x)$ при настолько большом значении $x^{\prime}$ их аргумента $x$, что выполнялись условия

$$
|| \frac{a\left(x^{\prime}\right)}{a\left(x^{\prime}+10\right)}|-1|<10^{-10}, \quad|| \frac{r_{\mathrm{eff}}\left(x^{\prime}\right)}{r_{\mathrm{eff}}\left(x^{\prime}+10\right)}|-1|<10^{-10} .
$$

Далее вычислялись отношения $\alpha(x)$ и $\beta(x)$, определенные как

$$
\alpha(x) \equiv \frac{a(x)}{a\left(x^{\prime}\right)}, \quad \beta(x) \equiv \frac{r_{\mathrm{eff}}(x)}{r_{\mathrm{eff}}\left(x^{\prime}\right)} .
$$

Все вычисления были выполнены при $x^{\prime}=1000$ для $2 \lambda=-1,1,3$. 
ТАБлицА 1. Безразмерные длины $a$ и эффективные радиусы $r_{\text {eff }}$ синглетного двумерного $n p$-рассеяния.

\begin{tabular}{|r|c|c|}
\hline $2 \lambda$ & $a$ & $r_{\text {eff }}$ \\
\hline-1 & -0.7114 & 5.7076 \\
\hline 1 & -2.5616 & 3.5035 \\
\hline 3 & -1.4595 & 12.023 \\
\hline
\end{tabular}

Перед обсуждением результатов вычислений следует пояснить физический смысл отношений (52). Значения функций $a(x)$ и $r_{\text {eff }}(x)$ в произвольно выбранной точке $x=b$ являются соответственно длиной рассеяния $a$ и эффективным радиусом $r_{\text {eff }}$ в случае потенциала $V(x)$, “обрезанного” в этой точке. Поэтому функции $\alpha(x)$ и $\beta(x)$ обладают прозрачным физическим смыслом: в той же точке $x=b$ значения этих функций являются относительными вкладами от воздействия потенциала $V(x)$ в выбранной области его аргумента $0 \leqslant x \leqslant b$ в соответствующие значения длины рассеяния $a$ и эффективного радиуса $r_{\text {eff. }}$. Следовательно, значения функций $\alpha(x)$ и $\beta(x)$ позволяют ответить на следующий физически важный вопрос: в какой области аргумента $x$ вклад потенциала $V(x)$ в длину рассеяния $a$ и эффективный радиус $r_{\mathrm{eff}}$ является пренебрежимо малым или, наоборот, определяющим?

Обсудим результаты вычислений. Найденные значения длин рассеяния и эффективных радиусов собраны в табл. 1 .

Поведение функций $a(x)$ и $r_{\text {eff }}(x)$ в случае $2 \lambda=-1$ иллюстрирует рис. 3. Функция $a(x)$ имеет нуль первого порядка в точке $x=x_{0}=1.285 \ldots$ В этой же точке функция $r_{\text {eff }}(x)$ терпит разрыв второго рода. Вследствие этой особенности вклад от потенциала $V(x)$, включенного на интервале $0<x<x_{0}$, в значение длины рассеяния $a$ равен нулю. Графики функций $a(x)$ и $r_{\text {eff }}(x)$ весьма близки к своим горизонтальным асимптотам $a(\infty) \equiv-0.7114 \ldots$ и $r_{\mathrm{eff}}(\infty)=5.7076 \ldots$ при сравнительно малом значении $x=x_{0} \approx 4$ их аргумента $x$. Следовательно, в обсуждаемом случае $2 \lambda=-1$ на интервале $x>x_{0}$ вклад от потенциала $V(x)$ в значения $a$ и $r_{\text {eff }}$ длины рассеяния и эффективного радиуса невелик.

На рис. 4 изображены графики функций $\alpha(x)$ и $\beta(x)$, вычисленные по формулам (52) в случаях $2 \lambda=1,3$. В обоих случаях эти графики близки к их горизонтальным асимптотам $\alpha(\infty)=1$ и $\beta(\infty)=1$ в области $x>x_{1} \approx 15$ довольно больших значений аргумента $x$. Кроме того, значения функций $\alpha(x)$ и $\beta(x)$ принадлежат соответственно интервалам $(0.5,1)$ и $(-0.5,1)$, если их аргумент изменяется на интервале $(6,15)$. Следовательно, в рассмотренных случаях $2 \lambda=1,3$ на этом интервале вклад от потенциала $V(x)$ в значения $a$ и $r_{\text {eff }}$ длин рассеяния и эффективных радиусов является определяющим.

Теперь отметим несколько особо значимых фактов. Потенциал Рида (51) является типичным представителем класса ядерных потенциалов, отталкивающих в области малых расстояний между ядрами и притягивающих в области больших расстояний. Следовательно, результаты выполненных расчетов, представленных выше в табл. 1 и на рис. 3 и 4, являются численным доказательством двух утверждений. Сформулируем их. 

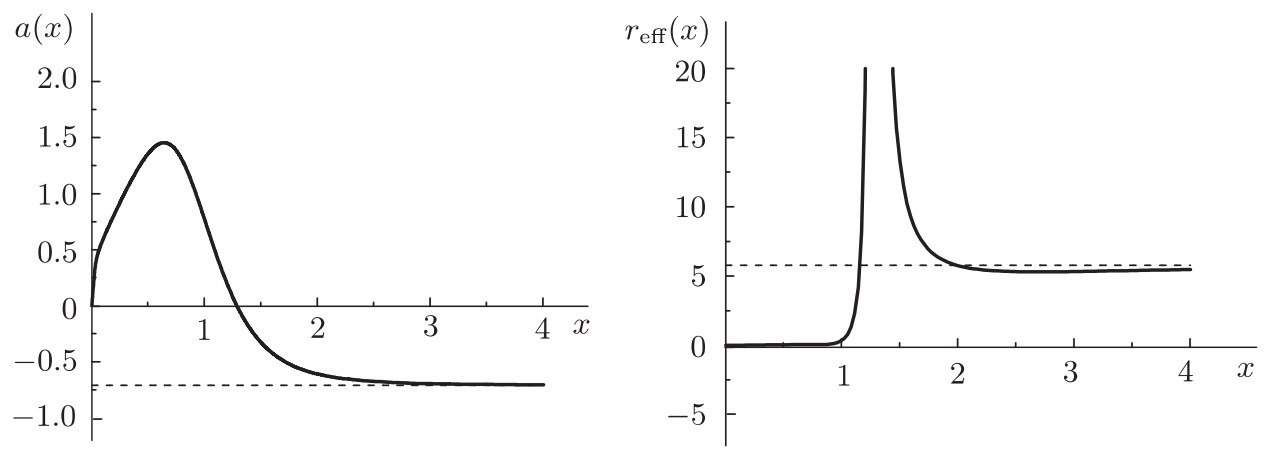

Рис. 3. Графики функций $a(x)$ и $r_{\text {eff }}(x)$ в случае $2 \lambda=-1$ (сплошные кривые) и их горизонтальные асимптоты $f_{a}(x) \equiv a(\infty)=-0.7114 \ldots$ и $f_{r}(x) \equiv r_{\mathrm{eff}}(\infty)=$ 5.7076 . . (штриховые прямые).
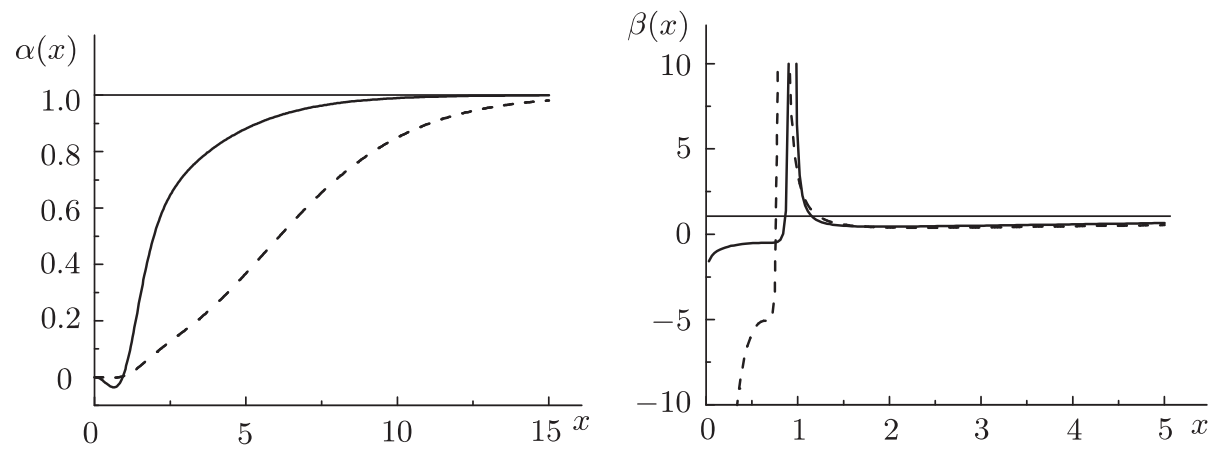

Рис. 4. Относительные вклады $\alpha(x)$ и $\beta(x)$, определенные формулами (52) в случае $2 \lambda=1$ (сплошные кривые) и в случае $2 \lambda=3$ (штриховые кривые); тонкая прямая - функция $f(x) \equiv 1$.

Предлагаемый подход, который основан на нелинейных уравнениях (17) или (18) и интегральном представлении (25), является экономичным с вычислительной точки зрения методом. Этот метод позволяет не только рассчитать с высокой точностью коэффициенты $a$ и $r_{\text {eff }}$, но и выявить прозрачным с физической точки зрения способом области расстояний, в которых вклад потенциала в такие коэффициенты является определяющим или, наоборот, несущественным.

Покажем, что все пять известных к настоящему времени методов вычисления параметров $a$ и $r_{\text {eff }}$ двумерного низкоэнергетического рассеяния менее эффективны по сравнению с нашим подходом.

Один из таких методов, основанный на рекуррентных системах линейных уравнений (28), (30) и (33), (35), (37), уже обсуждался в разделе 3.

Второй метод детально пояснен в работе [16]. Этот метод примени́м только в случае $2 \lambda=-1$ и реализуется по следующей схеме. Длиной рассеяния $a^{\prime}$ и эффективным радиусом $r_{\text {eff }}^{\prime}$ считаются коэффициенты асимптотики (8). В исходном уравне- 
нии Шредингера (2) полагается $\lambda=-1 / 2$ и $q=0$. Затем вычисляется регулярное в начальной точке $x=0$ решение $\tilde{u}(x)$ получившегося уравнения. Следующий этап заключается в последовательном вычислении двух интегралов

$$
X_{1} \equiv \int_{0}^{\infty} d x \sqrt{x} V(x) \tilde{u}(x), \quad X_{2} \equiv \int_{0}^{\infty} d x \sqrt{x} \ln x V(x) \tilde{u}(x),
$$

и далее длина рассеяния $a^{\prime}$, функция $v(x)$ и эффективный радиус $r_{\text {eff }}^{\prime}$ находятся по формулам

$$
a^{\prime}=e^{\left(X_{2}-1\right) / X_{1}}, \quad v(x)=\sqrt{x} \ln \frac{x}{a^{\prime}}, \quad r_{\text {eff }}^{\prime}=2 \int_{0}^{\infty} d x\left[v^{2}(x)-\tilde{u}^{2}(x)\right] .
$$

Третий, четвертый и пятый методы вычисления коэффициентов $a$ и $r_{\text {eff }}$ реализуются по одной и той же схеме: сначала вычисляются значения фазы рассеяния $\delta_{\lambda}(q)$ при нескольких достаточно малых положительных значениях $q_{i}, i=1,2, \ldots, n+1$, волнового числа $q$, затем найденные значения $\delta_{\lambda}\left(q_{i}\right)$ используются для экстраполяции функции эффективного радиуса (9) в точку $q=0$.

В третьем методе [1], [3] для определения значения $\delta_{\lambda}\left(q_{i}\right)$ фазы рассеяния используется асимптотика (4) предварительно вычисленного решения $u_{\lambda}\left(x, q_{i}\right)$ задачи Шредингера (2)-(4).

В четвертом методе [5] ключевым является нелинейное уравнение (15). В этом уравнении полагается $m=\lambda+1 / 2$ и $q=q_{i}$. Получившееся уравнение численно интегрируется на конечном отрезке $\left[0, x_{\max }\right]$ достаточно большой длины $\left(x_{\max } \gg 1\right)$, а затем значение $\delta_{\lambda}\left(q_{i}\right)$ фазы рассеяния определяется по формуле

$$
\delta_{\lambda}\left(q_{i}\right) \sim \operatorname{arctg} T\left(x_{\max } ; q_{i}\right)
$$

Пятый метод [20], [21] основан на системе линейных уравнений для амплитудных функций $c(x ; q)$ и $s(x ; q)$, подчиненных в начальной точке $x=0$ граничным условиям $c(x ; q)=1, s(x ; q)=0$. Следуя работе [21], используем определения (7), (12)-(14) функций $h(q), j_{\lambda}, n_{\lambda}$, запишем систему для амплитудных функций в виде

$$
\begin{aligned}
& \partial_{r} c(x ; q)=-\frac{1}{q} V(x)\left[c(x ; q) j_{\lambda}(\rho)-s(x ; q) n_{\lambda}(\rho)\right] n_{\lambda}(\rho), \\
& \partial_{x} s(x ; q)=-\frac{1}{q} V(x)\left[c(x ; q) j_{\lambda}(\rho)-s(x ; q) n_{\lambda}(\rho)\right] j_{\lambda}(\rho),
\end{aligned}
$$

и выразим тангенс фазы рассеяния через эти функции:

$$
\operatorname{tg} \delta_{\lambda}(q)=\lim _{x \rightarrow \infty} \operatorname{tg} \delta_{\lambda}(x ; q), \quad \operatorname{tg} \delta_{\lambda}(x ; q) \equiv \frac{s(x ; q)}{c(x ; q)+h(q) s(x ; q)} .
$$

Во всех трех упомянутых выше методах для нахождения коэффициентов $a$ и $r_{\text {eff }}$ по предварительно вычисленным значениям $\delta_{\lambda}\left(q_{i}\right), i=1,2, \ldots, n+1$, фазы рассеяния $\delta_{\lambda}(q)$ приходится известным в вычислительной математике способом [30] решать задачу экстраполяции таблично заданной функции полиномом конечной степени. Сформулируем эту задачу следующим образом: используя определение (9) функции эффективного радиуса $K(q)$ и ее представление в виде бесконечного ряда по 
четным степеням аргумента $q$, требуется вычислить коэффициенты $a$ и $r_{\text {eff }}$ низкоэнергетической асимптотики (11) этой функции.

Опишем метод решения поставленной задачи. Сначала по формуле (9) вычисляются все значения $K\left(q_{i}\right), i=1,2, \ldots, n+1$, функции $K(q)$, отвечающие известным значениям $\delta_{\lambda}\left(q_{i}\right)$ фазы рассеяния $\delta_{\lambda}(q)$. Затем функция $K(q)$ аппроксимируется полиномом $K_{n}(q)$ таким, что

$$
K_{n}(q)=\sum_{i=1}^{n+1} K_{i} q^{2(i-1)}, \quad K_{i} \equiv K\left(q_{i}\right), \quad i=1,2, \ldots, n+1
$$

Далее этот полином и его вторая производная вычисляются в точке $q=0$. В итоге для коэффициентов $a$ и $r_{\text {eff }}$ получаются следующие экстраполяционные приближения:

$$
a \approx-\frac{1}{K_{n}(0)}=-\frac{1}{K_{1}},\left.\quad r_{\mathrm{eff}} \approx \partial_{q}^{2} K_{n}(q)\right|_{q=0}=2 K_{2} .
$$

Как известно [30], точность экстраполяционных приближений улучшается с ростом числа известных значений аппроксимируемой функции. Поэтому для вычисления длины рассеяния $a$ и эффективного радиуса $r_{\text {eff }}$ третьим, четвертым или пятым методами приходится неоднократно интегрировать уравнения (2), (15) или (53). Следовательно, эти методы менее экономичны, чем предлагаемый подход, в котором для вычисления коэффициентов $a$ и $r_{\text {eff }}$ с высокой точностью и при любом значении квантового числа $\lambda$ достаточно один раз численно решить уравнение (17) или уравнение (18), а затем вычислить один интеграл (25).

\section{6. ОПРЕДЕЛЕНИЯ ДЛИНЫ РАССЕЯНИЯ И ЭФФЕКТИВНОГО РАДИУСА В ОСОБЫХ СЛУЧАЯХ}

Обсудим ряд (10) и его низкоэнергетическую (при $q \rightarrow+0$ ) асимптотику (11) в бесконечно удаленной точке $x=\infty$. При длине рассеяния $a=0$ первое слагаемое суммы (11) неограничено, и поэтому сумма не является низкоэнергетической асимптотикой ряда (10) при $x=\infty$. В случае бесконечно большой по модулю длины рассеяния (при $a= \pm \infty$ ) первое слагаемое обсуждаемой суммы равно нулю, а второе будет неограниченным по модулю, если $r_{\text {eff }}= \pm \infty$. Следовательно, и в этом случае ряд (10) в точке $x=\infty$ имеет иную асимптотику.

Для анализа двух указанных выше случаев нам потребуются известные представления [21] функций $K(x ; q), a(x), r_{\text {eff }}(x)$ и $P(x)$ через компоненты $c_{n}(x)$ и $s_{n}(x)$, а также основные свойства этих компонент [21]. На всей полуоси $x>0$ функция $K(x ; q)$ представляет собой дробь,

$$
K(x ; q)=\tau \frac{c_{0}(x)+q^{2} c_{1}(x)+q^{4} c_{2}(x)+\cdots}{s_{0}(x)+q^{2} s_{1}(x)+q^{4} s_{2}(x)+\cdots},
$$

функции $a(x)$ и $r_{\mathrm{eff}}(x)$ определяются формулами $(26)$ и $(31)$, а функция $P(x)$ задается как

$$
P(x)=-\frac{1}{r_{\mathrm{eff}}^{3}(x)} \frac{1}{2 s_{0}(x)}\left(2 \tau c_{2}(x)-s_{2}(x) \frac{c_{0}(x)}{s_{0}(x)}-s_{1}(x) r_{\mathrm{eff}}(x)\right)
$$


причем компоненты $c_{0}(x)$ и $s_{0}(x)$ не имеют общих нулей. Перечислим важные следствия указанного свойства компонент $c_{0}(x)$ и $s_{0}(x)$.

Пусть в некоторой точке $x=b>0$ компонента $s_{0}(x)$ имеет нуль первого порядка. Тогда в этой точке компонента $c_{0}(x)$ отлична от нуля, и в силу равенств $(26)$ и $(31)$ функция $a(x)$ имеет нуль первого порядка, а функция $r_{\text {eff }}(x)$ имеет полюс первого или второго порядка, если соответственно $s_{1}(b)=0$ или $s_{1}(b) \neq 0$. Согласно рис. 1a, рис. 16 и формулам (38)-(40) для притягивающего потенциала прямоугольной формы первый случай реализуется при $2 \lambda=-1$, а второй - при $2 \lambda \geqslant 1$. Теперь предположим, что некоторая точка $x=b>0$ является нулем первого порядка компоненты $c_{0}(x)$. Тогда в этой точке компонента $s_{0}(x)$ отлична от нуля и вследствие соотношений $(26)$ и (31) функция $a(x)$ имеет полюс первого порядка, а функция $r_{\text {eff }}(x)$ принимает конечное ненулевое или нулевое значение. Как следует из рис. 1в и формул (42), для отталкивающего потенциала прямоугольной формы второй случай имеет место при $2 \lambda=-1$.

Приступим к анализу особых случаев $a=0$ и $a= \pm \infty$. Для сокращения записи предельные при $x \rightarrow \infty$ значения используемых функций $K(x ; q)$ и $K^{ \pm}(x ; q)$ будем обозначать как $K(q)$ и $K^{ \pm}(q)$, а значения всех компонент $c_{n}(x), s_{n}(x)$ и функций $a(x), r_{\mathrm{eff}}(x), a^{ \pm}(x), r_{\mathrm{eff}}^{ \pm}(x)$ в точке $x=\infty$ - соответственно символами $c_{n}, s_{n}$ и $a, r_{\mathrm{eff}}$, $a^{ \pm}, r_{\text {eff }}^{ \pm}$.

Начнем с первого случая. Пусть $a=0$. Тогда согласно равенству (26) имеем $s_{0}=0$, но $c_{0} \neq 0$. Предположим, что $s_{1} \neq 0$. В области $x \gg 1$ введем функцию

$$
K^{+}(x ; q) \equiv \tau \frac{c_{0}(x)+q^{2} c_{1}(x)+q^{4} c_{2}(x)+\cdots}{q^{2} s_{1}(x)+q^{4} s_{2}(x)+\cdots}=-\frac{1}{a^{+}(x)}+\frac{q^{2}}{2} r_{\mathrm{eff}}^{+}(x)+\cdots,
$$

где по определению

$$
a^{+}(x) \equiv-\frac{s_{1}(x)}{\tau c_{0}(x)}, \quad r_{\mathrm{eff}}^{+}(x) \equiv \frac{2 \tau}{s_{1}(x)}\left(c_{1}(x)-s_{2}(x) \frac{c_{0}(x)}{s_{1}(x)}\right) .
$$

Перейдем к пределу $x \rightarrow \infty$. В этом пределе благодаря равенству $s_{0}=0$ из представлений (54) и (56) следует связь $q^{2} K^{+}(q)=K(q)$, а формулы $(26),(31),(55)$ и (57) порождают соотношения

$$
\begin{aligned}
a^{+} & \equiv \lim _{x \rightarrow \infty} a^{+}(x)=\frac{1}{2} \lim _{x \rightarrow \infty}\left[a^{2}(x) r_{\mathrm{eff}}(x)\right], \\
r_{\mathrm{eff}}^{+} & \equiv \lim _{x \rightarrow \infty} r_{\mathrm{eff}}^{+}(x)=2 \lim _{x \rightarrow \infty} \frac{a(x)-4 r_{\mathrm{eff}}(x) P(x)}{a^{2}(x)} .
\end{aligned}
$$

Используя определение (11) функции эффективного радиуса $K(q)$ через фазу рассеяния $\delta_{\lambda}(q)$, связь $q^{2} K^{+}(q)=K(q)$ и равенство (56), взятое при $x=0$, получаем искомые представления:

$$
K^{+}(q) \equiv q^{2 \lambda+3}\left[\operatorname{ctg} \delta_{\lambda}(q)-h(q)\right]=-\frac{1}{a^{+}}+\frac{q^{2}}{2} r_{\mathrm{eff}}^{+}+\cdots .
$$

Эти представления означают, что в рассмотренном случае $a=0$ вместо функции эффективного радиуса $K(q)$, нулевой длины рассеяния $a$ и эффективного радиуса $r_{\text {eff }}$ следует использовать функцию эффективного радиуса $K^{+}(q)$, ненулевую 
длину рассеяния $a^{+}$и конечный эффективный радиус $r_{\mathrm{eff}}^{+}$, определенные формулами (58) и (59).

Теперь исследуем второй особый случай. Положим $|a|=\infty$. Тогда из равенства $(26)$ следует, что $c_{0}=0$, но $s_{0} \neq 0$. Предположим, что $c_{1} \neq 0$. В области $x \gg 1$ введем функцию

$$
K^{-}(x ; q) \equiv \tau \frac{q^{2} c_{1}(x)+q^{4} c_{2}(x)+\cdots}{s_{0}(x)+q^{2} s_{1}(x)+q^{4} s_{2}(x)+\cdots}=-\frac{1}{a^{-}(x)}+\frac{q^{2}}{2} r_{\mathrm{eff}}^{-}(x)+\cdots,
$$

а функции $a^{-}(x)$ и $r_{\mathrm{eff}}^{-}$определим формулами

$$
a^{-}(x) \equiv-\frac{s_{0}(x)}{\tau c_{1}(x)}, \quad r_{\mathrm{eff}}^{-}(x) \equiv \frac{2 \tau}{s_{0}(x)}\left(c_{2}(x)-s_{1}(x) \frac{c_{1}(x)}{s_{0}(x)}\right) .
$$

В пределе $x \rightarrow \infty$ благодаря равенству $c_{0}=0$ представления (54) и (61) порождают связь $q^{-2} K^{-}(q)=K(q)$, а из формул $(26),(31),(55)$ и (62) следуют соотношения

$$
a^{-}=-\frac{2}{r_{\mathrm{eff}}}, \quad r_{\mathrm{eff}}^{-}=r_{\mathrm{eff}}^{3} P
$$

Используя определение (11) функции $K(q)$, связь $q^{-2} K^{-}(q)=K(q)$ и равенство (61), взятое при $x=\infty$, получаем искомые представления:

$$
K^{-}(q) \equiv q^{2 \lambda-1}\left[\operatorname{ctg} \delta_{\lambda}(q)-h(q)\right]=-\frac{1}{a^{-}}+\frac{q^{2}}{2} r_{\text {eff }}^{-}+\cdots
$$

Согласно этим представлениям в рассмотренном случае $|a|=\infty$ вместо функции эффективного радиуса $K(q)$, бесконечно большой по модулю длины рассеяния $a$ и ненулевого эффективного радиуса $r_{\text {eff }}$ следует использовать функцию эффективного радиуса $K^{-}(q)$, конечную и ненулевую длину рассеяния $a^{-}$и конечный эффективный радиус $r_{\text {eff }}^{-}$, определенные формулами (63).

\section{7. ЗАКЛЮЧЕНИЕ}

Суммируем основные результаты настоящей работы.

В разделе 3 нелинейная версия метода фазовых функций расширена на случай двумерного рассеяния квантовой частицы центральным короткодействующим потенциалом. Расширение заключается в выводе и качественном анализе новых уравнений (16)-(24), предназначенных для вычисления функции эффективного радиуса, длины рассеяния и эффективного радиуса.

В разделе 4 получены точные решения (38)-(44) уравнений (17)-(21) в случае потенциала прямоугольной формы и исследована зависимость длины рассеяния и эффективного радиуса от ширины такого потенциала.

В разделе 5 впервые вычислены длина и эффективный радиус двумерного рассеяния нейтрона на протоне в случае потенциала Рида с мягким кором. Для вычисления использованы уравнения (17), (18) и интегральное представление (25). Показано, что такой подход является наиболее экономичным и информативным по сравнению со всеми известными методами вычисления длины и эффективного радиуса двумерного рассеяния. 
В разделе 6 исследованы два особых случая $a=0,|a|=\infty$ и в этих случаях предложены новые определения (58)-(60) и (63), (64) функции эффективного радиуса $K^{ \pm}(q)$, длины рассеяния $a^{ \pm}$и эффективного радиуса $r_{\mathrm{eff}}^{ \pm}$. Следует отметить, что уравнения (16)-(24), их точные решения (38)-(44) и представления (58), (59) и (63) параметров $a^{ \pm}$и $r_{\mathrm{eff}}^{ \pm}$получены для любого значения квантового числа $\lambda$.

Поясним возможное применение перечисленных выше результатов. Вычислив длину рассеяния и эффективный радиус и используя затем асимптотику (11), мы можем найти низкоэнергетическое приближение фазы $\delta_{\lambda}(q)$, а затем по формуле (5) определить соответствующее приближение сечения $\sigma_{\lambda}(q)$. Анализ таких приближений дан в работе [21].

Используя известные длину рассеяния и эффективный радиус, с помощью метода, предложенного в работе [25], можно вычислить энергии слабосвязанных и околопороговых состояний квантовой частицы, движущейся в двумерной плоскости в поле центрального короткодействующего потенциала. Найденные длина и эффективный радиус позволяют по формулам, впервые полученным в работе [26], построить низкоэнергетические асимптотики всех парциальных сечений $\sigma_{\lambda}(q)$ при наличии таких состояний.

Представления (38)-(44) длины рассеяния и эффективного радиуса через функции Бесселя в случае потенциала прямоугольной формы и соответствующие таким представлениям приближения парциальных фаз и сечений предлагается использовать в теоретической физике ультрахолодных газов для моделирования упругого двумерного рассеяния в системе двух атомов или молекул. Полученные для данного потенциала представления (45)-(50) компонент $c_{n}(x)$ и $s_{n}(x)$ через функции Бесселя позволяют на основе известного метода [21] найти явную низкоэнергетическую асимптотику радиальной волновой функции $u_{\lambda}(x ; q)$ двумерного относительного движения двух атомов или молекул.

\section{Список литературы}

[1] Л. Д. Ландау, Е. М. Лифшиц, Теоретическая физика, т. 3: Квантовая механика. Нерелятивистская теория, Наука, М., 2004.

[2] В. де Альфаро, Т. Редже, Потенииалъное рассеяние, Мир, М., 1966.

[3] Дж. Тейлор, Теория рассеяния, Мир, М., 1975.

[4] Ф. Калоджеро, Метод фазовых функиий в теории потенииального рассеяния, Мир, M., 1976.

[5] В. В. Бабиков, Метод фазовых функиий в квантовой механике, Наука, М., 1976.

[6] Дж. Е. Браун, А. Д. Джексон, Нуклон-нуклонные взаимодействия, Энергоатомиздат, M., 1979.

[7] H. A. Bethe, Phys. Rev., 76:1 (1949), 38-50.

[8] G. Modugno, F. Ferlanio, R. Heidemann, G. Roati, M. Inguscio, Phys. Rev. A., 68 (2003), 011601, 4 pp.

[9] P. Clade, C. Ryu, A. Ramanathan, K. Helmerson, W. D. Phillips, Phys. Rev. Lett., 102:17 (2009), 170401, 4 pp.

[10] L. D. Carr, D. DeMille, R. V. Krems, J. Ye, New J. Phys., 11 (2009), 055049, 87 pp.

[11] F. Serwane, G. Zürn, T. Lompe, T. B. Ottenstein, A. N. Wenz, S. Jochim, Science, 332:6027 (2011), 336-338.

[12] I. R. Lapidus, Amer. J. Phys., 50:1 (1982), 45-47. 
[13] I. R. Lapidus, Amer. J. Phys., 54:5 (1986), 459-461.

[14] B. J. Verhaar, J.P.H.W. van den Eijnde, M. A. J. Voermans, M. M. J. Schaffrath, J. Phys. A, 17:3 (1984), 595-598.

[15] B. J. Verhaar, L. P. H. de Goey, J.P.H.W. van den Eijnde, E. J. D. Vredenbregt, Phys. Rev. A., 32:3 (1985), 1424-1429.

[16] N. N. Khuri, A. Martin, J.-M. Rishard, T. T. Wu, J. Math. Phys., 50:7 (2009), 072105, 17 pp., arXiv: 0812.4054.

[17] D. Bollé, F. Gesztesy, Phys. Rev. Lett., 52:17 (1984), 1469-1472.

[18] D. Bollé, F. Gesztesy, Phys. Rev. A., 30:3 (1984), 1279-1293.

[19] S. K. Adhikari, W. G. Gibson, Phys. Rev. A, 46:7 (1992), 3967-3977.

[20] S. A. Rakityansky, N. Elander, J. Phys. A, 45:13 (2012), 135209, 28 pp., arXiv: 1201.0172.

[21] В. В. Пупышев, ЯФ, 77:5 (2014), 699-710.

[22] S. K. Adhikari, Amer. J. Phys., 54:4 (1986), 362-366.

[23] P. G. Averbuch, J. Phys. A, 19:12 (1986), 2325-2335.

[24] B. Simon, Ann. Phys., 97:2 (1976), 279-288.

[25] В. В. Пупышев, ТМФ, 179:1 (2014), 102-122.

[26] В. В. Пупышев, Приближение эффективного радиуса в задаче двумерного рассеяния квантовой частицы центральным короткодействуюшим потенииалом, Препринт Р4-2013-85, ОИЯИ, Дубна, 2013.

[27] Дж. Сансоне, Обыкновенные дифференииальные уравнения, т. 1, ИЛ, М., 1953.

[28] Q.-G. Lin, Amer. J. Phys., 65:10 (1997), 1007-1009.

[29] М. Абрамовиц, И. Стиган (ред.), Справочник по специальным функциям с формулами, графиками и математическиим таблицами, Наука, М., 1979.

[30] Ю. С. Завьялов, Б. И. Квасов, В. Л. Мирошниченко, Методы сплайн-функиий, Наука, M., 1980.

Поступила в редакцию 29.07.2013, после доработки 20.05.2014 